\title{
FAMOSS, a conserved 41-aa peptide involved in plant tip
} \section{growth regulation}

Anna Mamaeva ${ }^{1, *}$, Andrey Kniazev ${ }^{1}$, Ilia Sedlov ${ }^{1}$, Nina Golub ${ }^{1}$, Daria Kharlampieva ${ }^{2}$, Valentin Manuvera ${ }^{2}$, Victor Rakitin ${ }^{3}$, Alexander Nosov ${ }^{3}$, Artem Fomenkov ${ }^{3}$, Marat Pavlyukov ${ }^{1}$, Sergey Kovalchuk ${ }^{1}$, Rustam Ziganshin ${ }^{1}$, Anna Glushkevich ${ }^{1}$, Vassili Lazarev ${ }^{2,4}$, and Igor Fesenko $^{1}$

${ }^{1}$ The Laboratory of functional genomics and plant proteomics, Shemyakin and Ovchinnikov Institute of Bioorganic Chemistry of the Russian Academy of Sciences, Moscow, 117997, Russian Federation;

${ }^{2}$ Department of Cell Biology, Federal Research and Clinical Center of Physical-Chemical Medicine of Federal Medical Biological Agency, Moscow, 119435, Russian Federation;

3 Timiryazev Institute of Plant Physiology, Russian Academy of Sciences, 127276 Moscow, Russian Federation;

${ }^{4}$ Moscow Institute of Physics and Technology (National Research University), Dolgoprudny, Moscow region, 141701, Russian Federation.

Correspondence: * Anna Mamaeva (AnnetteSt@yandex.ru)

Running Title: lncRNA-encoded peptide regulates plant growth

\section{Abstract}

Recent evidence shows that small open reading frame (smORF; <100 codons)-encoded peptides (SEPs) containing transmembrane domains are preadapted to be progenitors of novel functional genes. A dozen of such SEPs translated from long non-coding RNAs (lncRNAs) are already functionally characterised in animals. However, functional plant lncRNA-smORF-coded peptides are not yet described. Here, we report detailed functional characterization of a 41-aa peptide encoded by lncRNA-smORFs in the moss Physcomitrium patens, which was named "FAst-growing MOSS" (FAMOSS). We found that the FAMOSS interacts with the Rab-type small GTPase proteins and its overexpression leads to faster moss growth rate and more intensive vesicular transport in apical cells, while its knockout results in the opposite effect. The FAMOSS contains a predicted transmembrane domain and possible orthologs from streptophyta algae to flowering plants have a very conserved structure. Thus, the FAMOSS peptide is a 
previously unknown conserved player of Rab-mediated processes in plants. Our findings are in line with functional studies of transmembrane SEPs in animals and prove the principles of SEPs evolution. This study provides new insights into functions of plant lncRNA-smORFs.

Keywords: peptide encoded by lncRNA-smORF, Physcomitrium patens, vesicular trafficking

\section{Introduction}

Eukaryotic transcriptomes contain numerous RNAs with low coding potential (non-coding RNAs), including long non-coding RNAs (lncRNAs), primary microRNAs (pri-miRNA), and circular RNAs (circRNAs) (Li et al., 2017). LncRNAs are transcripts longer than 200 nucleotides that contain only small open reading frames (smORFs; <100 codons) and are unlikely to encode functional proteins. Despite this definition, several functional peptides and microproteins have recently been shown to be encoded by smORFs in mice (Anderson et al., 2015), humans (D'Lima et al., 2017; Huang et al., 2017; Slavoff et al., 2014), zebrafish (Chng et al., 2013), fruit flies (Immarigeon et al., 2021; Magny et al., 2013), Arabidopsis thaliana (Hanada et al., 2013), and the moss Physcomitrium (previously Physcomitrella) patens (Fesenko et al., 2019). These smORF-encoded peptides (SEPs) are involved in the regulation of cell proliferation (Polycarpou-Schwarz et al., 2018), cancer growth (Guo et al., 2020; Huang et al., 2017), apoptosis and autophagosome formation (Rubtsova et al., 2018), mitochondrial respiratory chain activity (Chugunova et al., 2019), muscle function (Matsumoto et al., 2017), antigen presentation (Niu et al., 2020), heart development (Chng et al., 2013), and sexual reproduction (Immarigeon et al., 2021). Unlike for animals, only a few examples of functional SEPs have been described in plants. They have been shown to regulate plant programmed cell death (Blanvillain et al., 2011), root growth and development (Casson et al., 2002; Chen et al., 2020), cell proliferation and expansion (Brito et al., 2018; Ikeuchi et al., 2011), nodule formation (Rohrig et al., 2002), and pollen germination (Dong et al., 2013).

Because of their small size, smORFs do not contain functional domains or may include only simple ones, such as a transmembrane $\alpha$-helix. Indeed, a "transmembrane-first" model of gene birth suggests that peptides with a transmembrane domain may accidently appear in thymine-rich non-genic regions (Vakirlis et al., 2020). A pool of uncharacterized SEPs located at, and interacting with, cellular membranes was detected in different studies; for example, the 56-aa Mtln peptide interacts with NADH-dependent cytochrome b5 reductase (Chugunova et al., 2019), the 90-aa SPAR peptide interacts with V-ATPase (Matsumoto et al., 2017), and the 28-aa Sarcolamban A interacts with Sarco-endoplasmic Reticulum $\mathrm{Ca}^{2+}$ adenosine triphosphatase 
66

67

68

69

70

(SERCA) (Magny et al., 2013). The identification of protein partners of the SEPs helps elucidate their functions (Matsumoto et al., 2017; Slavoff et al., 2014); for example, a human 7-kDa microprotein, NoBody, binds the mRNA decapping complex to regulate the number of P-bodies in a cell (D'Lima et al., 2017). The 10-kDa microprotein CASIMO1 controls cell proliferation by interacting with squalene epoxidase that is a key enzyme in cholesterol synthesis and a known oncogene in breast cancer (Polycarpou-Schwarz et al., 2018).

Previously, we identified functional smORFs encoded by lncRNAs in the model plant Physcomitrium patens, a moss (Fesenko et al., 2019). The knockout of four selected SEPs, PSEP1, PSEP3, PSEP18, and PSEP25, resulted in significantly reduced growth rates in the moss protonemata (Fesenko et al., 2019). In this study, we investigated the functions of PSEP1, which was renamed the FAst-growing MOSS (FAMOSS) peptide. We demonstrated that this SEP participates in the regulation of polar tip growth in P. patens via its interaction with Rab-type small GTPases. We observed that mutant plants lacking FAMOSS had altered apex-derived vesicular trafficking and different protonemal filament structures compared with the wild type. Our quantitative proteomic analysis revealed a downregulation of the stress-responsive proteins in both the FAMOSS-overexpressing (OE) and knockout (KO) lines, as well as an altered sensitivity to phytohormones. Finally, we proposed that the FAMOSS peptide is a novel component of the Rab protein complexes that regulate vesicular trafficking and stress responses in plants.

\section{Results}

\section{FAMOSS is widely distributed across different plant lineages}

Recently, we used a combined approach to identify functional SEPs encoded by lncRNAs in the model plant $P$. patens (Fesenko et al., 2019). In this analysis, we identified the 41-aa peptide PSEP1 (Physcomitrella smORF-encoded peptide 1), encoded by the transcript CNT2064811, which was predicted to be a non-coding RNA in the CANTATAdb database (Szczesniak et al., 2016) but was missed in the Phytozome V12 annotation (Goodstein et al., 2012; Lang et al., 2018). The overexpression of this peptide resulted in larger moss plant sizes and a faster growth rate, whereas the KO mutant had the opposite phenotype (Fesenko et al., 2019). Based on the observed phenotypic effects, we renamed this peptide "FAMOSS" here.

The inspection of the genome region around CNT2064811 revealed that the protein-coding gene Pp3c9_20610V3 is located in close proximity to this lncRNA. To exclude the possibility 
that the FAMOSS peptide is an upstream or downstream smORF located within annotated mRNA, we additionally performed direct nanopore RNA sequencing (ONT DRS) on the protonemal and gametophore transcriptomes (Fesenko et al., 2021). ONT DRS allows the accurate assembly and identification of the exon-intron structure of the transcripts. This analysis confirmed that CNT2064811 is an independent transcriptional unit with a higher transcriptional

103 level than the surrounding protein-coding genes (Figure 1A). To additionally explore whether

104 FAMOSS is translated from the predicted SmORF, we used CRISPR-Cas9 to fuse a reporter $105 G U S$ sequence to the FAMOSS sequence in the moss genome (Figure 1B-D). We observed that 106 FAMOSS expression both in the protonemata and gametophores was consistent with the results of the transcriptomic analysis.

We next analyzed the conservation of the FAMOSS peptide across different plant lineages.

109 Using a peptide amino acid sequence as a query, we performed a TBLASTN search (E-value 110 cutoff < 0.001) against the transcriptomes from the 1000 plants project (OneKP) and manually 111 inspected the results. This search revealed possible orthologs in the liverworts, other mosses, and 112 ferns, suggesting that this peptide was widely conserved in certain lineages (Figure 1E, F); 113 however, no hits could be detected in the hornworts, lycophytes, or vascular plants. In addition, 114 an ortholog of the FAMOSS peptide was also found in the transcriptome of Zygnemopsis sp. 115 (streptophyte algae). Although the C-terminal end of the FAMOSS peptide was poorly 116 conserved, the analysis of multiple alignments between the possible orthologs in green algae, 117 mosses, liverworts, and ferns revealed a pattern of highly conserved amino acid residues (Figure 118 1E). To identify potential conserved motifs in the FAMOSS peptide, the MEME algorithm 119 (Bailey et al., 2015) was used. This approach confirmed the presence of a significantly conserved motif, spanning the first 30 aa of the FAMOSS peptide (Supplemental Figure 1).

Given that the identification of peptide homologs can be problematic in distant species, we next performed a HMMER search $(E$-value cutoff $<0.01)$ against the translated transcriptomes

123 of selected Gymnospermae and Angiosperms species from the OneKP project (One Thousand 124 Plant Transcriptomes, 2019) and manually curated the results. This analysis revealed possible 125 orthologs in eight Gymnospermae species (12 transcripts, mean $=44$ aa), 108 Core 126 Eudicots/Rosids species (124 transcripts, mean = 47 aa), 41 Core Eudicots/Asterids species (53 127 transcripts, mean $=53 \mathrm{aa}$ ), and 25 Basal Eudicots (38 transcripts, mean $=46 \mathrm{aa})$ (Supplemental 128 Table 1); however, further studies are needed to confirm that these peptides are true orthologs. 129 The corresponding transcripts do not encode ORFs longer than 100 aa, and 50\% (63/124) of the 130 transcripts from the Core Eudicots/Rosids, 66\% (35/53) from the Core Eudicots/Asterids, and $13171 \%$ (27/38) from the Basal Eudicots were annotated as lncRNAs in a database of lncRNAs in 132 Angiosperms, AlnC (Singh et al., 2021). 
According to a prediction made using TMHMM version 2.0 (Krogh et al., 2001), the $P$.

134 patens FAMOSS peptide contains a transmembrane domain (Fesenko et al., 2021). To further

135 investigate the relationship between the conserved amino acid residues and the structural

136 properties of FAMOSS, we predicted the $P$. patens FAMOSS 3D structure using a recently

137 developed machine learning algorithm, AlphaFold2 (Jumper et al., 2021), which was shown to

138 have promising results. According to the obtained structural model, the N-terminal and central

139 parts of the peptide represent an alpha-helix with a slight kink in its center, probably associated with

140 a proline residue. The $\mathrm{C}$ terminus was predicted to be disordered (Figure 1G). To further explore

141 this finding, we analyzed the structures of predicted FAMOSS orthologs from five other plants

142 from different taxonomic groups (algae, liverworts, ferns, conifers, and dicots), revealing similar

143 results overall (Supplemental Figure 2). Our findings suggest that an ortholog of the FAMOSS

144 peptide was present in the last common ancestor of land plants and streptophyte algae and widely

145 conserved in different land plant lineages. The high expression level of the corresponding

146 transcript suggests that this peptide is an unannotated highly conserved small protein. 
A

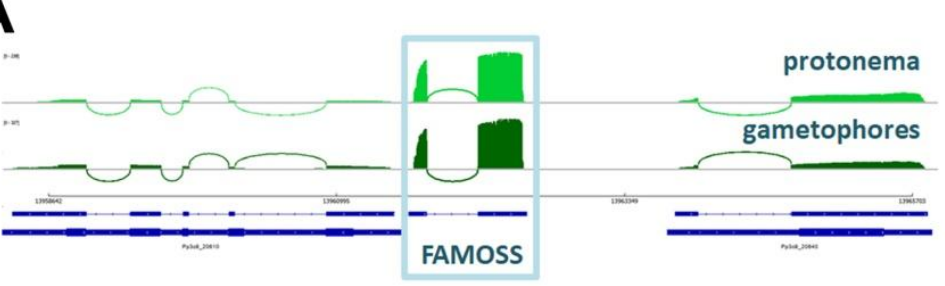

B

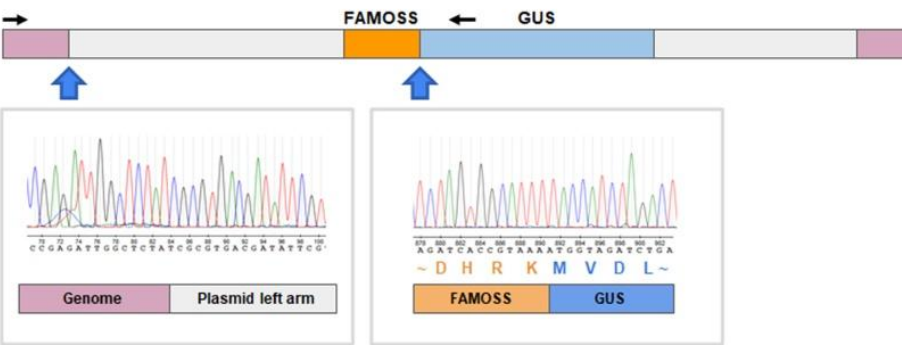

E

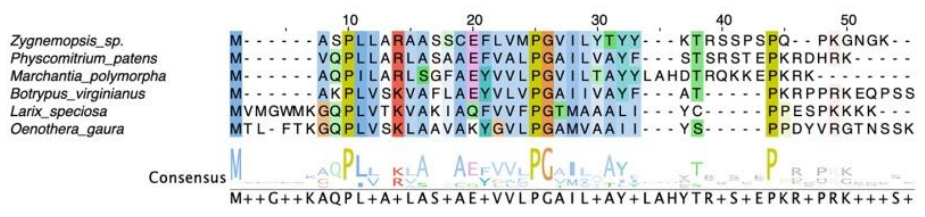

$\mathbf{F}$

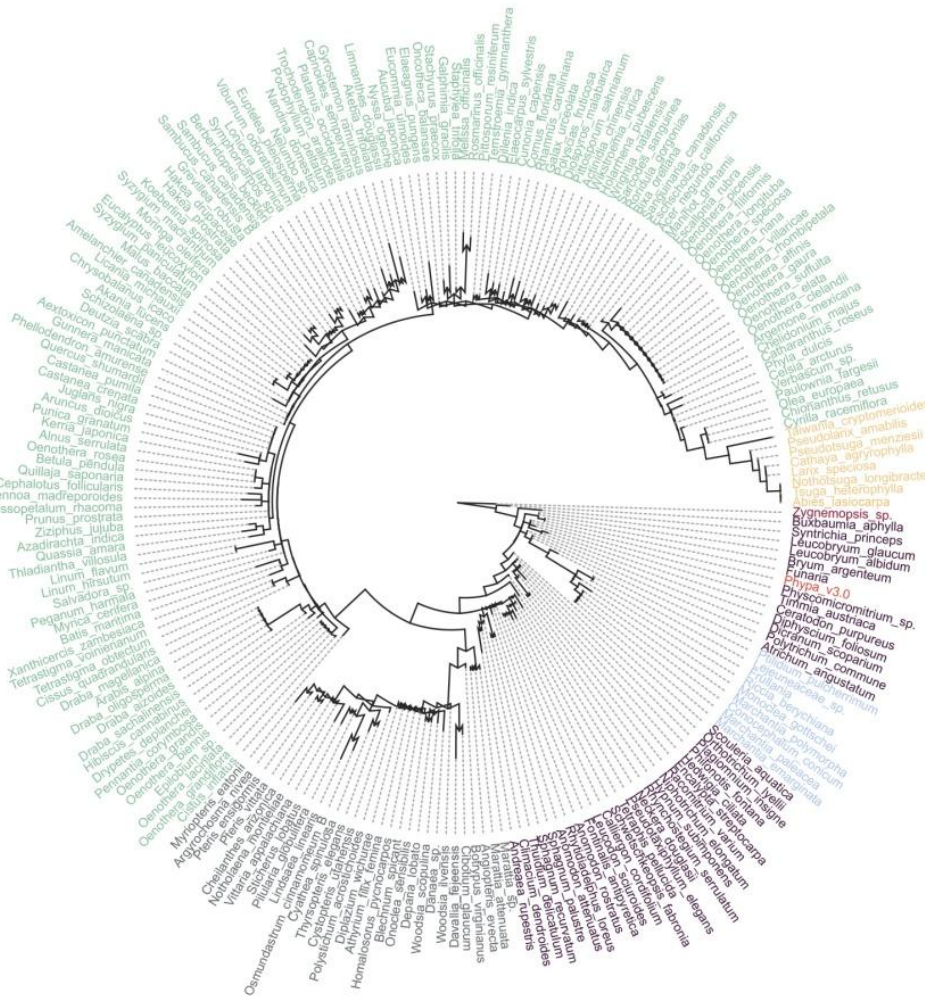

Figure 1. FAMOSS is a widely conserved plant smORF-encoded peptide.

151 sequencing in Physcomitrium patens protonema and gametophores. B - Construction of 152 FAMOSS coupled with a GUS reporter protein at the FAMOSS promoter. Black arrows indicate 
153 primers used for identification. C, D - GUS staining of the protonema $(\mathrm{C}$, scale bar $=200 \mu \mathrm{m})$

154 and gametophores ( $\mathrm{D}$, scale bar $=1 \mathrm{~mm})$. E - The alignment of the FAMOSS peptide and orthologous sequences from different plants. F - Phylogenomic tree of the identified FAMOSS orthologs. Mosses, burgundy; liverworts, blue; algae, red; ferns, gray; gymnosperms, orange; angiosperms, green. G - P. patens FAMOSS structure and structures of its orthologs, predicted using AlphaFold.

\section{FAMOSS regulates the tip growth rate in protonemata}

Previously, we showed that $P$. patens FAMOSS OE mutant lines displayed a rapid protonemal growth phenotype, whereas the KO lines grew more slowly than the wild-type plants (Fesenko et al., 2019). Moss protonemata consist of two morphologically distinguishable cell types, chloronemata and caulonemata (Figure 2A). Chloronemata are produced first, as the spores germinate, while caulonemal cells develop several days later at the tips of the protonemata (Vidali and Bezanilla, 2012). Caulonemal cells are characterized by their higher growth rate than chloronemal cells and tangential cell walls (Jang and Dolan, 2011).

To test the hypothesis that the rapid growth of the FAMOSS OE lines is due to the accumulation of caulonemal filaments, the proportion of chloronemal to caulonemal cells was compared in the wild-type and mutant lines. The ratio of caulonemal to chloronemal cells was significantly higher in the OE line and lower in the KO line when compared with the wild type (Figure 2A; $n=3$ independent biological repeats; chi-square test, $P<10^{-15}$ ). The chloronemal cells therefore predominate in the KO line (Supplemental Figure 3), suggesting a link between FAMOSS expression and the transition from chloronemal to caulonemal cells in P. patens. We next compared the length of the apical and two subapical cells between the wild-type and mutant lines. Although the lengths of the apical cells were similar in all genotypes, the subapical cells were significantly longer in the $\mathrm{OE}$ line than in the wild type or the $\mathrm{KO}$ line (Figure $2 \mathrm{~B}$, ANOVA with post-hoc Tukey HSD (Honestly Significant Difference) $P<0.00001$ ). These observations led to the hypothesis that the observed changes in the moss growth rate come from an altered protonemal structure (caulonemata/chloronemata ratio) and increased subapical cell lengths.

The chloronemal-to-caulonemal transition is regulated by both hormonal crosstalk and 183 different factors, such as glucose, light, and low nutrient availability (Jaeger and Moody, 2021). 184 Low light, cytokinins, and abscisic acid (ABA) are negative regulators of caulonemal 
185 differentiation, whereas auxin, glucose, and a high light intensity are positive regulators. We

186 therefore investigated how both negative and positive regulators influence the caulonemal 187 transition in the wild-type and mutant lines (Fig. 2C-F).

188 First, we tested the influence of cytokinin and ABA treatments on the growth rate of both 189 mutant lines (Figure 2C, D). It has previously been shown that large concentrations of cytokinins 190 in the growth medium inhibit filament growth and induce callus-like structures (Sabovljevic et 191 al., 2014). In our experiments, filament growth in the wild-type and KO plants was strongly 192 inhibited under a $0.1 \mu \mathrm{M}$ 6-benzylaminopurine (BAP) treatment, but the FAMOSS OE mutant 193 line produced long protonemal filaments, suggesting a decreased sensitivity to cytokinins (Figure 194 2C, E). Another negative regulator, ABA, significantly inhibited the growth of both the wild195 type and KO moss (Figure 2D and F; ANOVA with post-hoc Tukey HSD $P<0.001$ ), but there 196 was no effect on the growth rate of the OE line. Thus, the overexpression of FAMOSS led to a 197 reduced sensitivity to the negative regulators of moss protonemal growth, cytokinin, and ABA.

198 Ammonium tartrate (AT) is known to reduce the transition into caulonemal cells and 199 promote chloronemal branching (Vidali and Bezanilla, 2012). Given the altered ratios of 200 chloronemal to caulonemal filaments in the mutant lines, we compared the growth of the 201 different genotypes on culture mediums with and without AT. The application of AT 202 significantly reduced the growth rates of both the wild-type and mutant plants $(P<0.01$; Figure 203 2G), although both mutant lines significantly differed from the wild type, with the OE plants 204 being the smallest. On the medium without AT, the OE plants showed the fastest growth, but the 205 KO plants grew more slowly than the other genotypes (Figure 2G). 
$\mathbf{A}$

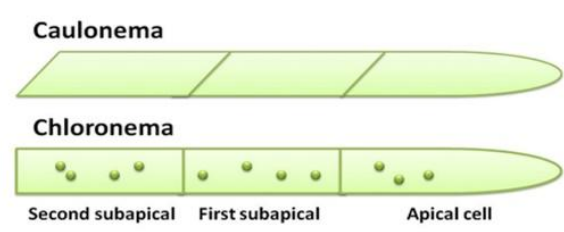

Type \%, caulonema \%, chloronema Ratio Caul/Chlor

\begin{tabular}{l|l|l|l|}
\hline WT & $59.72 \%$ & $40.28 \%$ & 1.5 \\
\hline OE & $76.73 \%$ & $23.27 \%$ & 3.3 \\
\hline KO & $33.48 \%$ & $66.52 \%$ & 0.5 \\
\hline
\end{tabular}

C

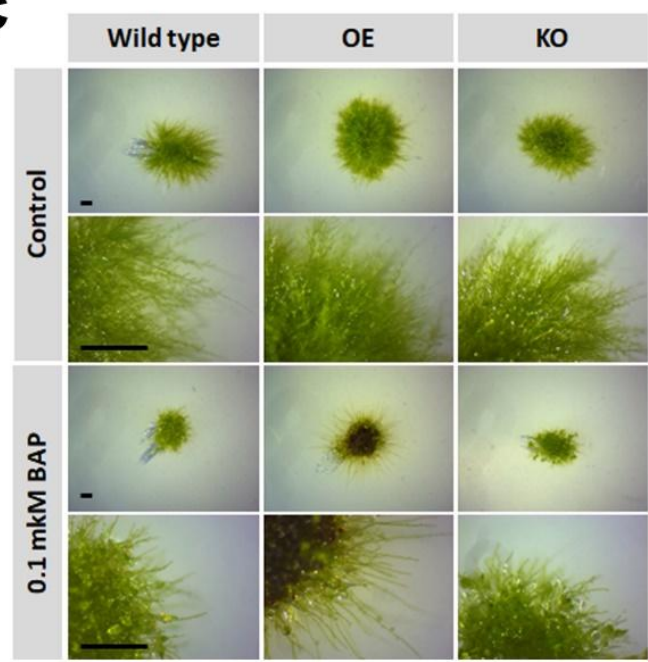

B

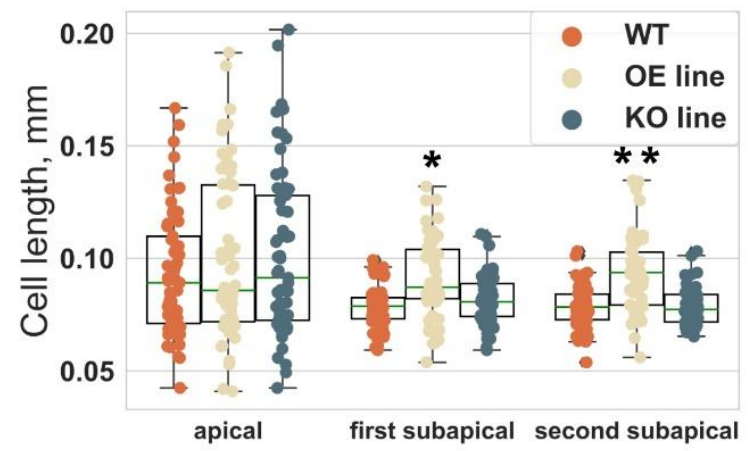

D

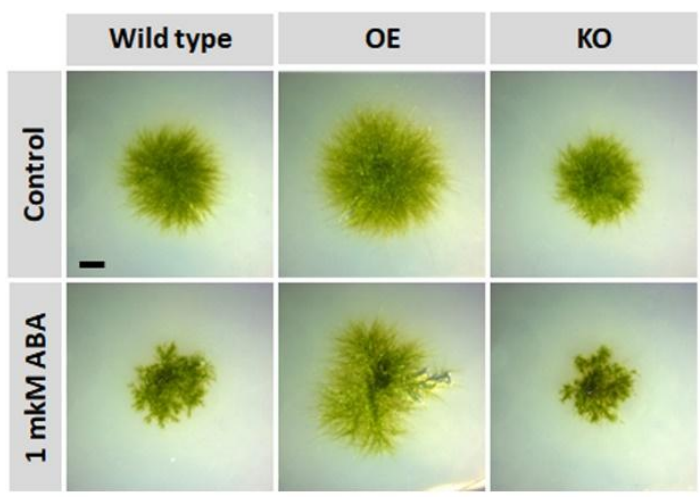

$\mathbf{E}$

$\mathbf{F}$

G

H
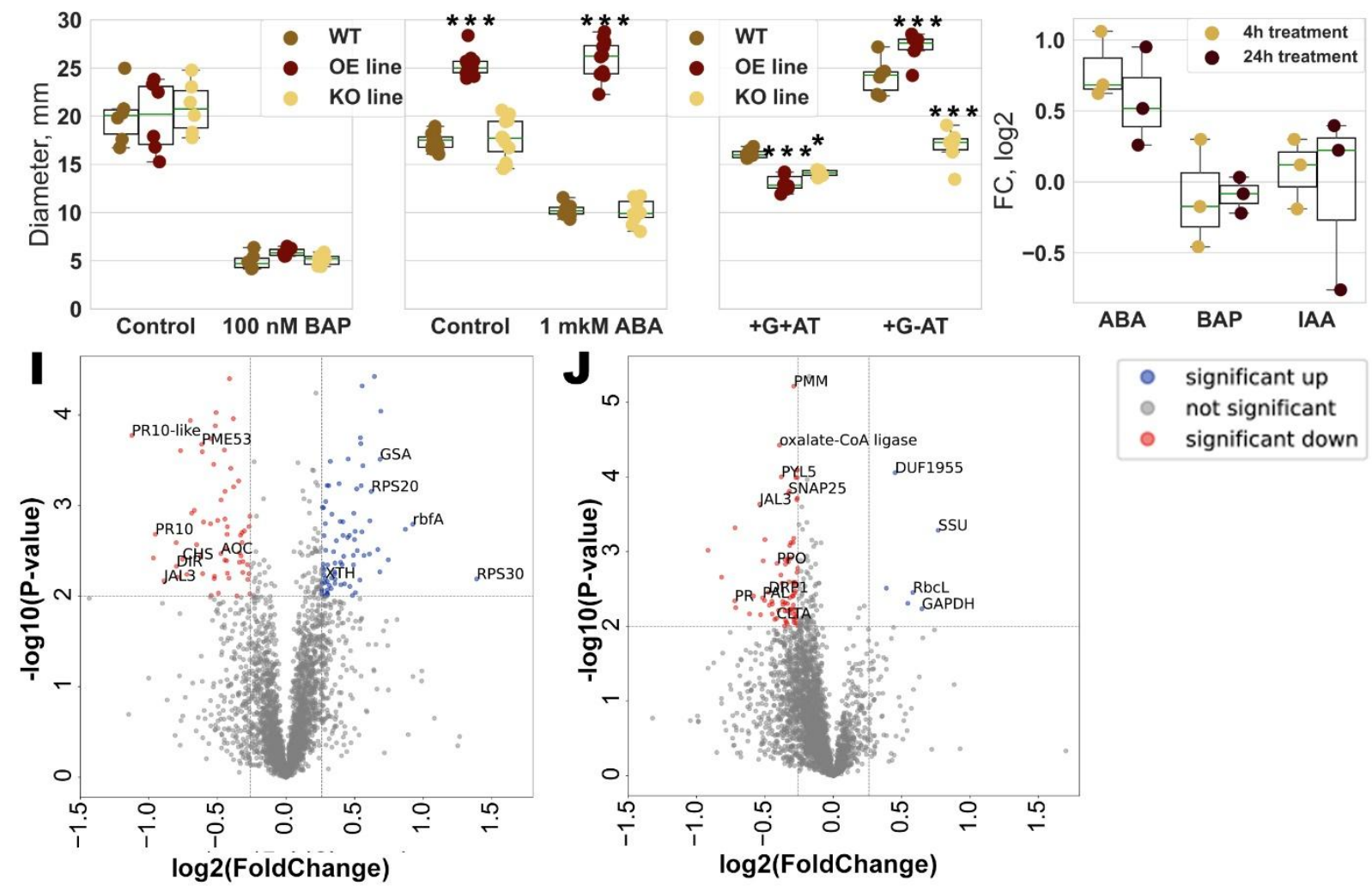

- significant up

- not significant

- significant down

Figure 2. The growth of mutant lines in different conditions.

A - The ratios of the two types of moss protonema filaments, caulonemata and 209 chloronemata, in wild-type, FAMOSS-overexpressing (OE), and knockout (KO) plants. B - The 
210 lengths of the apical, first subapical, and second subapical cells. C - The effect of 6-

211 benzylaminopurine (BAP) on protonemal morphology. Scale bar $=1 \mathrm{~mm}$. D - The effect of

212 abscisic acid (ABA) on protonemal morphology. E - The effect of BAP on the protonemal

213 growth rate. $\mathrm{F}$ - The effect of $\mathrm{ABA}$ on protonemal growth. $\mathrm{G}$ - The effect of medium

214 composition on protonemal growth. +G+AT, BCD medium supplemented with glucose and

215 ammonium tartrate; +G-AT, BCD medium with glucose but without ammonium tartrate. $\mathrm{H}-$

216 Fold change (FC) in FAMOSS transcription after treatments with ABA, BAP, and indole-3-acetic

217 acid (IAA). I, J - Quantitative proteomic analysis of the FAMOSS-overexpressing (OE) and

218 knockout $(\mathrm{KO})$ plants. Volcano plots of the entire set of proteins quantified in the iTRAQ

219 analysis in the FAMOSS OE (I) and KO (J) lines. Proteins with significant differences in

220 abundance relative to the wild type are depicted in color. GSA, glutamate-1-semialdehyde 2,1-

221 aminomutase; rbfA, ribosome-binding factor A; RPS20, ribosomal protein S20; RPS30,

222 ribosomal protein S30; AOC, allene oxide cyclase; GST, glutathione $S$-transferase; CHS,

223 chalcone synthase; XTH, xyloglucan endotransglucosylase/hydrolase protein 23; PME, pectin

224 methylesterase; rbcL, ribulose-1,5-bisphosphate carboxylase/oxygenase large subunit; GAPDH,

225 glyceraldehyde-3-phosphate dehydrogenase A chloroplastic-like isoform X2; JAL3, jacalin-

226 related lectin 3; PPO, polyphenol oxidase; PAL, phenylalanine ammonia-lyase; PR10,

227 pathogenesis-related protein; PR10-like, pathogenesis-related protein-like; DIR, dirigent protein;

228 SNAP25, synaptosomal-associated protein 25; ASPL, tether containing UBX domain for

229 GLUT4; DRP1, dynamin-related protein 1E; CLTA, clathrin light chain; PYL5, pyrobactin

230 resistance 1-like 5; PMM, phosphomannomutase; SSU, ribulose-1,5-bisphosphate

231 carboxylase/oxygenase small subunits; DUF1955, domain of unknown function.

*, $P<0.05 ; * *, P<0.01 ; * * *, P<0.001$.

The stimulatory effect of auxin on the transition from chloronemal to caulonemal filaments has been shown previously; however, exogenous auxin treatments resulted in the inhibition of moss growth (Thelander et al., 2018). In our experiments, the growth of wild-type moss plants was significantly inhibited under auxin treatment starting with $1 \mathrm{nM}$ indole-3-acetic acid (IAA; Supplemental Figure 4). In contrast, the growth rate of the OE line was significantly decreased only by $1 \mu \mathrm{M}$ IAA (ANOVA with post-hoc Tukey HSD $P<0.001$ ). As the auxin concentration increased, the protonemal filaments were increasingly composed of caulonemata, resulting in a sparser and lighter appearance. Thus, FAMOSS is expressed in conditions favorable to caulonemata formation, causing chloronemata to differentiate into caulonemata, which increases

243 the protonemal growth rate. 
We next tested whether the auxin and cytokinin treatments influenced the transcriptional level of FAMOSS; however, no difference was observed after a 4- or 24-h treatment with either phytohormone (Figure 2H). By contrast, FAMOSS expression was marginally upregulated by ABA (two-tailed t-test, $P<0.05$ ). Our findings showed that the overexpression of FAMOSS positively regulates protonemal growth and the transition from chloronemal to caulonemal filaments. In particular, sensitivity to the hormonal negative regulators of the caulonemal transition was reduced in the OE line. In addition, the proportion of caulonemal filaments was reduced in the KO plants, which had a similar phenotype to the wild-type plants, although the growth rate was lower on the medium without the inhibitor of caulonemal growth (AT).

\section{Proteomic analysis of the mutant lines}

We next used isobaric tags for relative and absolute quantitation (iTRAQ)-based comparative quantitative proteomic analyses to identify changes in the proteomes of the mutant lines. Because iTRAQ quantification underestimates the amount of real fold change (Ow et al., 2009), fold change ratios of $>1.20$ or $<0.83$ ( $P<0.01$, one-way ANOVA) were used to identify differentially expressed protein groups (DEPs). Overall, we identified 118 DEPs in the OE line and 75 DEPs in the KO line (Figure 2I, J; Supplemental Table 2).

The most upregulated DEPs in the OE line were ribosomal proteins, such as Pp3c2_29010 (RP-S30e), Pp3c24_17080 (RP-S20), and Pp3c12_16410 (rbfA) (Figure 2I). Among the most downregulated DEPs in the OE line, we identified a group of proteins that participate in the stress response; for example, the pathogenesis-related proteins (PR-10) Pp3c2_27350 and Pp3c7_19850 were previously shown to be involved in the defense response (Castro et al., 2016) and downregulated under osmotic stress (Stevenson et al., 2016). In addition, proteins related to the biotic stress response were downregulated, including Pp3c5_22560 (dirigent protein; DIR), Pp3c5_22560 (chalcone synthase; CHS), and Pp3c4_22490 (allene oxide cyclase; AOC). Stressinduced DIR plays a role in the adaptive response (Paniagua et al., 2017), while CHS is a key enzyme of the flavonoid/isoflavonoid biosynthesis pathway and is involved in the salicylic acid biosynthesis pathway (Dao et al., 2011). AOC is involved in the jasmonic acid biosynthesis pathway (Stenzel et al., 2003). Another protein related to secondary metabolism regulation and

272 the defense response is Pp3c9_1620 (polyphenol oxidase; PPO) (Tran et al., 2012). The protein pectin methylesterase (Pp3c5_12660) was also downregulated in the OE line. Pectin 
276 to a variety of stress conditions. However, additional experiments are needed to confirm or 277 dismiss this hypothesis.

278 The proteome changes in the KO line were less pronounced (Figure 2J). The chloroplastic 279 proteins, such as the large and small subunits of RuBisCO and Pp3c11_15790 (glyceraldehyde 280 3-phosphate dehydrogenase (GAPDH)), were among the most upregulated in the KO line 281 (Supplemental Table 2). One possible explanation for this result is the predominance of 282 chloroplast-rich chloronemal filaments in this line. The stress-related proteins were also 283 downregulated in the $\mathrm{KO}$ line, as was seen in the proteome of the OE line; for example, 284 Pp3c7_19850 (PR10), Pp3c6_6545 (DIR-related), and Pp3c1_18940 (phenylalanine ammonia285 lyase; PAL) were downregulated. It is worth noting that Pp3c7_19850 (PR10), Pp3c18_10760 (glutamine synthetase), Pp3c9_1620 (polyphenol oxidase), and Pp3c1_1940 (jacalin-like lectin domain) were downregulated in both the OE and the KO lines. The top 10 most downregulated proteins included two proteins containing the cupin (Cupin_2) domain (Supplemental Table 2). Proteins with this domain play crucial roles in plant development and defense (Wang et al., 2014).

We also identified a group of downregulated DEPs associated with vesicle trafficking in the KO line. These were dynamin-related protein 1C (DRP1C, Pp3c19_4870), which regulates vesicle formation in post-Golgi trafficking (Fujimoto and Tsutsumi, 2014; Jilly et al., 2018); clathrin light chain (Pp3c7_10220; CTLA); and synaptosomal-associated protein-related (Pp3c2_7850; SNAP25). SNAP25 is a SNARE (soluble $N$-ethylmaleimide-sensitive factor attachment protein receptor) protein, which is involved not only in vesicular trafficking under normal conditions but also in regulating transport of cell wall-associated and defense proteins during biotic stress (Kwon et al., 2020). SNARE-mediated membrane trafficking is also required for the proper response of plants to abiotic stresses.

300 The iTRAQ analysis thus showed that proteins involved in the response to different stress conditions were downregulated in both mutant lines. By contrast, the most upregulated DEPs in

302 the OE line belong to biosynthetic processes, suggesting that the rapid growth rate in the OE line 303 is linked to proteome changes.

\section{The stress response is altered in the mutant lines}

According to the quantitative proteomic analysis, some stress-related proteins were downregulated in both mutant lines, suggesting that they may have altered stress tolerances. In abiotic stress factors. 
A

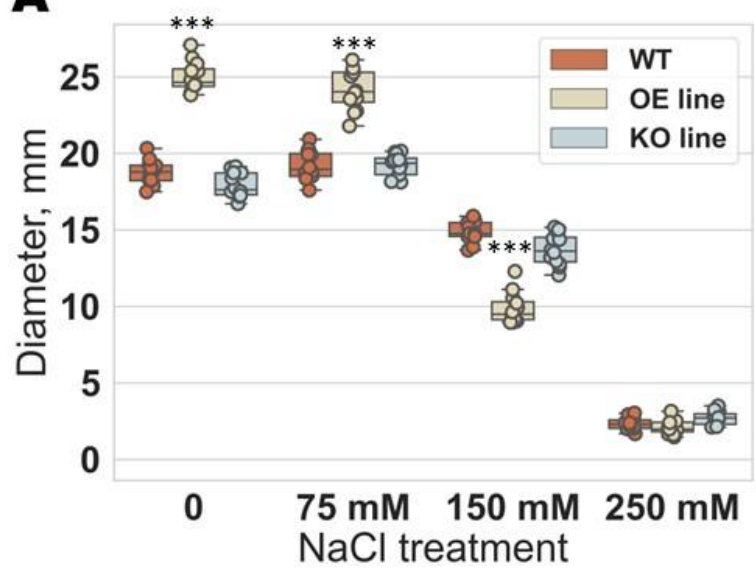

C

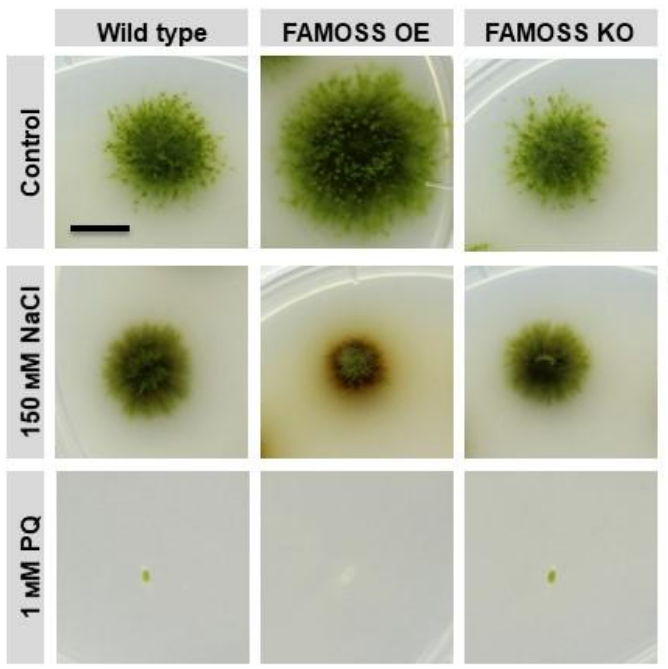

B

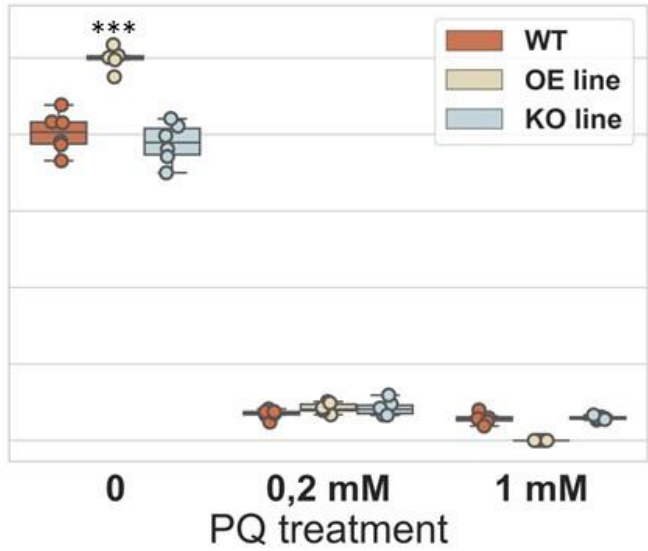

D

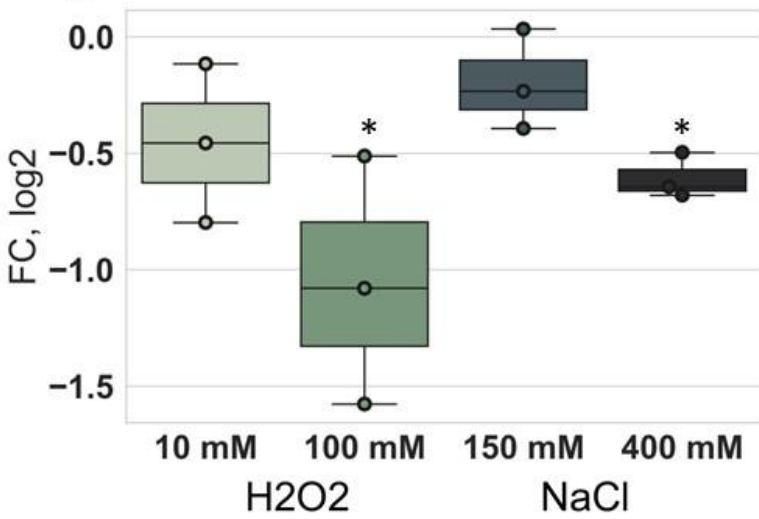

Figure 3. The growth rate of the mutant lines under stress-related conditions

311 A - Effect of $\mathrm{NaCl}$ on protonemal growth. B - Effect of paraquat (PQ) on protonemal growth. C -

312 Effect of $\mathrm{NaCl}$ and PQ on protonemal morphology after 30 days of cultivation. D - FAMOSS

313 expression under different stresses. * $P<0.05$; ***, $P<0.001$.

We next investigated the growth of both the mutant and wild-type plants under abiotic stress conditions induced by salt $(\mathrm{NaCl})$ and paraquat $(\mathrm{PQ})$ (Figure 3A-C). All genotypes were severely affected starting with the $150 \mathrm{mM} \mathrm{NaCl}$ concentration (Figure 3A); however, the growth rate of the $\mathrm{OE}$ plants was significantly reduced compared with the wild type and $\mathrm{KO}$ under this concentration of $\mathrm{NaCl}(P<0.001$, ANOVA with post-hoc Tukey HSD test).

319 Moreover, the OE line had the most severely altered phenotype after the $150 \mathrm{mM} \mathrm{NaCl}$ treatment (Figure 3C).

PQ is a potent reactive oxygen species (ROS) inducer. The growth of all genotypes was significantly inhibited by a treatment with either 0.2 or $1 \mathrm{mM}$ PQ $(P<0.001$, ANOVA with 
323 post-hoc Tukey HSD test; Figure 3B, C). Moreover, the growth of the OE line was completely

324 inhibited and the plants died under $1 \mathrm{mM}$ PQ (Figure 3C).

Using RT-qPCR analysis, we found that the level of FAMOSS transcription in the wildtype protonemata was only marginally decreased after a 1-h treatment with $400 \mathrm{mM} \mathrm{NaCl}$ or 100 $\mathrm{mM} \mathrm{H} \mathrm{H}_{2} \mathrm{O}_{2}$ (two-tailed t-test, $P<0.05$; Figure 3D). This result suggests that FAMOSS is not stress-inducible at the transcriptional level.

Our results showed that the mutants, especially the OE line, had a reduced ability to adapt to stress. Given that ABA is the most important regulator of the abiotic stress response, the rapid protonemal growth and altered ABA sensitivity in the FAMOSS OE line could result in the observed phenotypes.

\section{FAMOSS interactome}

We further investigated the possible protein interaction partners of the FAMOSS peptide using two approaches, a pull-down assay and coimmunoprecipitation (co-IP) (Figure 4A-C). For the pull-down assay, a recombinant FAMOSS peptide fused with streptavidin at the N-terminal end (FAMOSS-SAV; see Materials and Methods) and recombinant streptavidin were used (Figure 4B). We used reversible cross-linking to bind the interacting proteins and identified them using a mass spectrometry (MS) analysis. This approach revealed 323 unique protein groups in the FAMOSS-SAV samples (Supplemental Table 3). Besides the large clusters of ribosomal and chloroplast proteins, which are common contaminants (Mellacheruvu et al., 2013), the most promising protein partners were Rab-type small GTPases (Figure 4D). Rab proteins participate in a diverse range of cellular processes, including vesicular trafficking, tip growth, and the stress response (Chen, Heo, 2018; Lycett, 2008; Ma, 2007). Because many contaminants were observed in the FAMOSS-SAV interactomes, we performed a gel electrophoresis analysis of the samples and analyzed the $25-\mathrm{kDa}$ band using an in-gel trypsin digestion followed by a liquid chromatography (LC)-MS/MS analysis to enrich the fraction of Rab proteins.

The final list of Rab-type GTPases, unique for FAMOSS-SAV samples, included RabD2B/Rab-1A, RabA2B/Rab-11A, RabF/Rab-5, RabA5A/Rab-11A, RabE/Rab-8A, and RabG/Rab-7A (Figure 4D; Supplemental Table 3). Among these proteins, the largest peptides coverage (33\%) and intensity were observed for the Pp3c14_15250 (RabA2B/Rab-11A) protein.

To validate the obtained results, we generated mutant lines overexpressing FAMOSSFLAG fusion constructs (Figure 4A). The FLAG tag was placed on the C terminus (mutant 1028 line) or on the $\mathrm{N}$ terminus (mutant 1032 line) of the FAMOSS peptide (Figure 4B). We 
355 immunoprecipitated FLAG-tagged FAMOSS and identified RabD2B/Rab-1A, RabA2B/Rab-

356 11A, RabA5A/Rab-11A, and RabE/Rab-8A in the interactome of the 1032 line $(n=3$

357 independent experiments) (Figure 4D). These proteins were unique for the FAMOSS-FLAG

358 interactomes and were not found in the control samples.

359 Due to high sequence similarity of Rab GTPases (Figure 4E; Lycett, 2008; Ma, 2007;

360 Minamino and Ueda, 2019), we cannot rule out the possibility that FAMOSS peptide interacts

361 with several Rab proteins. For example, human GDI1 protein was shown to interact with

362 different Rab GTPases: RAB3A, RAB5A, RAB5B, RAB5C, RAB8A, RAB8B, RAB10, RAB35

363 and RAB43 (Steger et al., 2017). In addition, due to similar subcellular localization (Elliott et al.,

364 2020) Rab proteins from different families could co-purify together in our experiments.

365 Thus, our results suggest that the Rab GTPase proteins may interact with the FAMOSS

366 peptide. The Rab GTPase proteins target vesicles to the plasma membranes and mediate their

367 fusion, thereby stimulating the intensity of vesicular trafficking to the cell apex and increasing

368 the tip growth rate (Lycett, 2008; Ma, 2007). This is consistent with the phenotype of the

369 FAMOSS OE lines. 
A

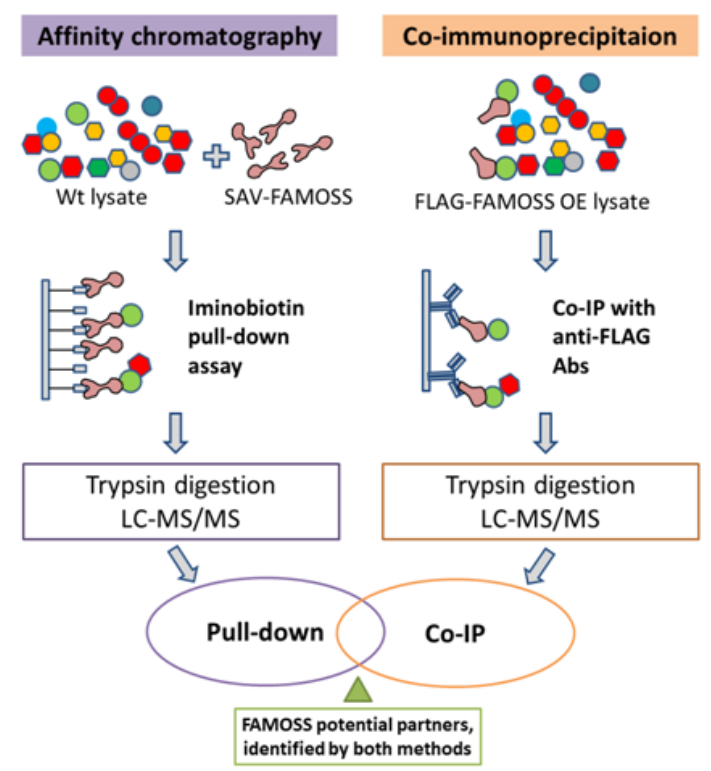

B FAMOSS with FLAG-tag on the $\mathrm{N}$-end MDYKDDDDKG GGGSGGGGMV QPLLARLASA AEFVALPGAI LVAYFSTSRS TEPKRDHRK

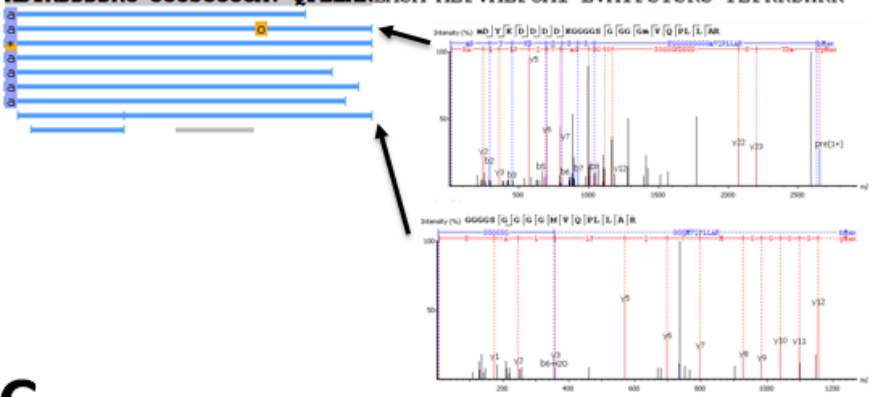

FAMOSS-SAV

MGSMRKIVVAAIAVSLTTVITASASADPSKDSKAQVSAAEAGITGTMYN QLGSTIIVTAGADGALTGTYESAVGNAESRYVLTGRYDSAPATDGSGTA LGWTVAWKNNYRNAHSATTWSGQYVGGAEARINTQWLLTSGTTEANA WKSTLVGHDTFTKVKKPSAASIDAAKKAGVNNGNPLDAVQQSRGGSGG GSENLYFQGVDMVQPLLARLASAAEFVALPGAILVAYFSTSRSTEPKRD HRK

D

\begin{tabular}{|c|c|c|}
\hline Subclass of Rab GTPases & Pulldown with FAMOSS-SAV & Coimmunoprecipitation \\
\hline RabD2B/Rab-1A & + & + \\
\hline RabA2B/Rab-11A & + & + \\
\hline RabF/Rab-5 & + & - \\
\hline RabA5A/Rab-11A & + & + \\
\hline RabE/Rab-8A & + & + \\
\hline RabG/Rab-7A & + & - \\
\hline
\end{tabular}

E

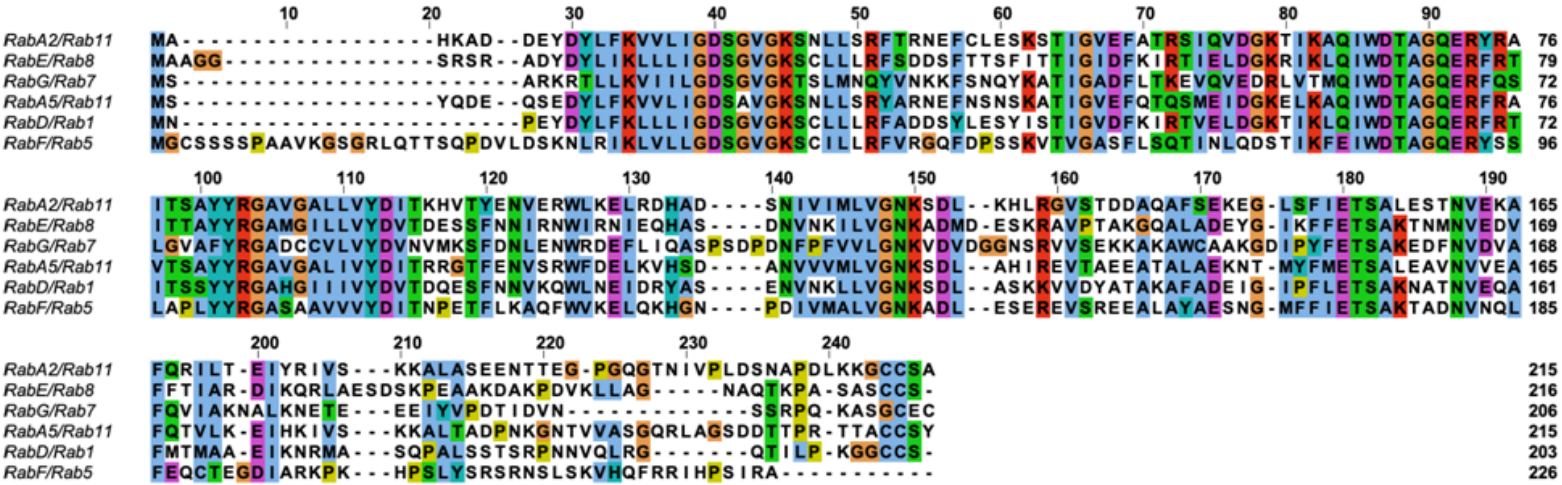

373 Figure 4. Analysis of the protein partners of the FAMOSS peptide. A - Diagram of the 374 interactome experiments. B - Sequence and coverage of the FLAG-tagged FAMOSS sequence.

375 C - Sequence of streptavidin (SAV)-tagged FAMOSS. D - Rab GTPases present in the two types 376 of interactome experiments. Proteins detected in samples are marked with a plus sign, and those 
377 not detected are indicated with a minus sign. E - Alignment of Rab GTPases found in the

378 FAMOSS interactomes.

\section{FAMOSS regulates vesicular trafficking}

The moss protonemal filaments expand by polarized tip cell growth similar to pollen tubes or root hairs in flowering plants (Orr et al., 2020; Rensing et al., 2020; Rounds and Bezanilla, 2013; Vidali and Bezanilla, 2012). It is well known that plant tip growth requires active apical vesicular trafficking regulated by different families of small GTPases, including Rab proteins (Minamino and Ueda, 2019). The RabA GTPases were previously shown to regulate polarized secretion and vesicle delivery in plants (Chen, Heo, 2018; de Graaf et al., 2005; MartiniEre and Moreau, 2020; Szumlanski and Nielsen, 2009).

To determine whether the FAMOSS peptide influences the vesicle pool in the apex cells, we labelled protonemal filaments of the wild type and both mutant lines using the fluorescent dye SynaptoGreen C4 (also known as FM1-43, a trademark of Molecular Probes) (Jelinkova et al., 2010) (Figure 5A-C). The FM1-43 dye is widely used for a marking of vesicles in the tip growing plant cells such as pollen tube, root hair and protonema apical cells (Samaj et al., 2005;

393 Bove et al., 2008; Pleskot et al., 2012; Gisbergen et al., 2020). In the wild-type cells, the 394 distribution of the apical vesicles was similar to that which was previously described in $P$. patens 395 (Rawat et al., 2017; Gisbergen et al., 2020) (Figure 5B); however, the labelling intensity was 396 significantly increased in the OE line compared with both the wild-type and KO lines (ANOVA, $397 \quad P<0.001$; Figure 5D, E). This suggests a more intensive vesicle trafficking in the cell apex, 398 which is consistent with the rapid protonemal growth in the OE line. By contrast, the 399 fluorescence intensity was significantly decreased in the cell apexes of the protonemal filaments 400 in the KO line compared with the wild-type and OE line cells (ANOVA, $P<0.001$; Figure 5E).

401 These results suggest that the FAMOSS peptide may function as a possible modulator of 402 vesicular trafficking during tip cell growth via its interaction with the Rab small GTPases. 

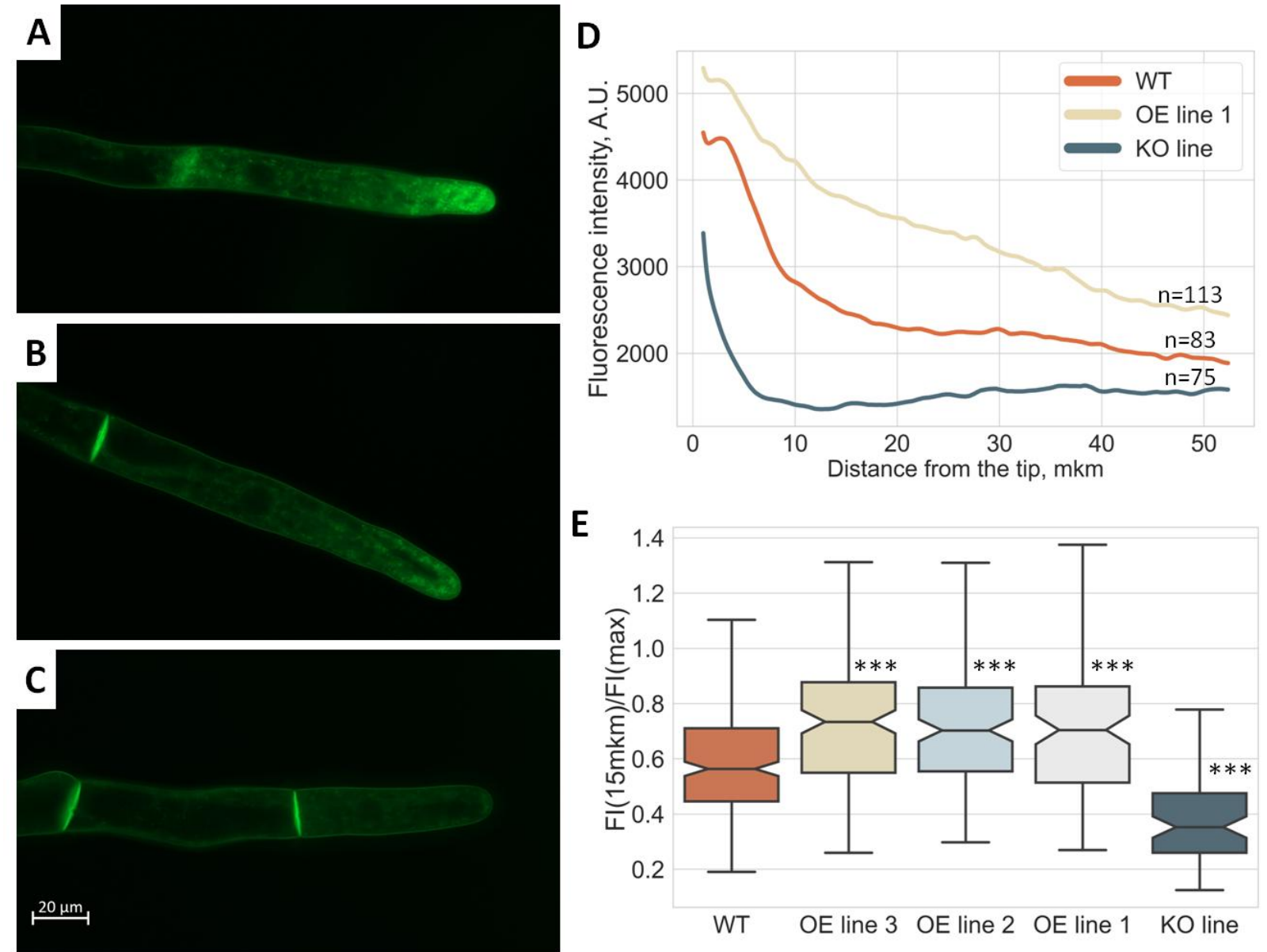

Figure 5. The FAMOSS peptide affects vesicular trafficking. A-C - Apical protonemal cells stained with SynaptoGreen C4. (A - FAMOSS-overexpression line (OE); B - The wild type; C - FAMOSS knockout line (KO). D - Profile of SynaptoGreen C4 fluorescence in the apical cells. E - Boxplot showing the ratios of intensity of SynaptoGreen C4 staining at the distance $15 \mathrm{mkm}$ from the cell tip to the maximal intensity. ***, $P<0.001$.

412 Given that apical vesicular transport is linked to cell wall formation (Rounds and Bezanilla,

413 2013), we next analyzed the regeneration of the protonemal filaments after protoplast isolation.

414 The proportion of regenerated plants with new chloronemal filaments was significantly higher in 415 the KO line $(\sim 18 \%)$ than in the wild type $(\sim 8 \%)$ or the OE line $\left(\sim 2 \%\right.$; chi-squared test $\left.P<10^{-10}\right)$.

416 The FAMOSS peptide therefore appears to inhibit the process of chloronemal formation, which 417 results in a more effective regeneration process in the KO line. This correlates with the observed 418 chloronemata/caulonemata ratio in the protonemal tissue. 


\section{Discussion}

A growing body of evidence suggests that the peptides or microproteins encoded by smORFs may play important roles in animal and plant cells (Casson et al., 2002; Huang et al., 2017; Matsumoto et al., 2017; Polycarpou-Schwarz et al., 2018; Rohrig et al., 2002; Slavoff et al., 2014; Tavormina et al., 2015); however, there are few examples of functional SEPs in plants. In our previous study, we identified a new 41-aa sORF-encoded peptide that influences moss growth located on a transcript predicted to be a lncRNA (Fesenko et al., 2019).

In this study, we identified possible FAMOSS orthologs in liverworts, mosses, ferns, conifers, and eudicots, indicating that it is widely conserved across the plant kingdom. A FAMOSS ortholog was also found in Zygnemopsis sp. (streptophyte algae). This is consistent with the findings that the Zygnematophyceae appear to be the closest relatives of land plants (Delwiche and Cooper, 2015; Nishiyama et al., 2018; One Thousand Plant Transcriptomes, 2019). The corresponding transcripts do not encode longer (>100 aa) proteins, suggesting that the FAMOSS orthologs can be considered the main ORFs. The conservation of the FAMOSS peptide across different plant lineages is consistent with our results, in which a set of Rab-type small GTPases were identified in the FAMOSS interactomes (Table 1). Rab GTPases are highly conserved regulators of membrane trafficking in plants (Lycett, 2008; Ma, 2007; Minamino and Ueda, 2019) and are present in both algae (Hoepflinger et al., 2013) and land plants (Minamino and Ueda, 2019). In particular, proteins from the RabA clade are involved in the coordination of tip cell growth (Ma, 2007); for example, these proteins are crucial for the tip-targeted vesicular trafficking of cell wall components in root hairs (Preuss et al., 2004) and pollen tubes (Szumlanski and Nielsen, 2009) in Arabidopsis. Tip growth of the moss protonemata is also regulated by the Rab proteins (Orr et al., 2021); thus, the consideration of Rab GTPases as possible partners of the FAMOSS peptide is consistent with the protonemal growth rates, subapical cell lengths, and chloronemata-to-caulonemata transition in the mutant lines. Moreover, using the fluorescent dye SynaptoGreen C4, we revealed changes in the intensity of the vesicular trafficking in the mutant lines. A previous study showed that the AtRabA1d protein accumulated in the apical dome of the growing root hairs in Arabidopsis, completely colocalizing with intracellular vesicles stained with the fluorescent dye FM4-64 (Ovecka et al., 2010). In addition, the quantitative proteomic analysis revealed that some proteins associated 
452 GTPases or with proteins from the corresponding protein complex may affect the intensity of

453 vesicular trafficking in the moss tip cells and influence the protonemal growth rate.

In addition to regulation of tip growth in plants, Rab proteins are also involved in phytohormone signaling and the stress response. AtRabE1c interacts with the ABA receptor PYL4 (pyrobactin resistance 1-like 5) to stimulate the ABA response and stress tolerance in Arabidopsis (Chen et al., 2021), while the overexpression of OsRab11 in rice (Oryza sativa) resulted in a decreased plant sensitivity to ABA and enhanced plant tolerance to salt and osmotic stresses (Chen, Heo, 2018). Here, we found that FAMOSS OE plants were less sensitive to ABA treatment and strongly inhibited by salt stress. Moreover, the stress-related proteins were downregulated in both the FAMOSS OE and KO lines, suggesting a link between the functions of the Rab proteins and FAMOSS expression levels.

In conclusion, we described the functions of a new plant SEP encoded by a lncRNA in the moss $P$. patens. We suggest that this peptide, named FAMOSS, is a possible interaction partner of the Rab GTPases, which in turn control numerous processes, including vesicular trafficking, polar tip growth, phytohormone signaling, and stress tolerance, processes that were altered in the FAMOSS OE/KO mutants. The FAMOSS peptide could therefore be considered an important component of Rab signaling in plants. Further studies will help elucidate the mechanisms of such interactions.

\section{Acknowledgments}

This research was supported by the Russian Science Foundation (project No. 17-1401189). We thank Igor Mazheika for the help with plant cultivation and good advices.

\section{Author contributions}

474 A.M. and I.F. conceived and designed experiments. A.M. and I.F. wrote the manuscript with 475 input from all authors. I.F. supervised the project. A.K and A.G. performed nanopore sequencing 476 and analyzed the sequencing data. A.M., S.K. and R.Z. performed the proteomics analysis. A.M., 477 N.G., I.S., V.R., A.N. and A.F. conducted analysis of phenotypes and fluorescent microscopy. 478 N.G. and A.K. analyzed transcription using RT-PCR. I.F. performed the statistical and 479 bioinformatics analyses. A.K., D.K., V.M. and V.L. created genetic construction, generate 480 recombinant proteins, and performed moss transformation experiments. I.S., M.P. and A.M. 481 performed pull-down and co-immunoprecipitation. All authors read and approved the final manuscript. 


\section{Declaration of Interests}

The authors declare no competing interests.

\section{Materials and Methods}

\section{Physcomitrium patens growth conditions}

Physcomitrium patens subsp. patens (“Gransden 2004”, Frieburg) protonemata of wild type and FAMOSS (PSEP1) - knockouting and overexpressing lines (Fesenko et al., 2019) were grown on BCD medium supplemented with $5 \mathrm{mM}$ ammonium tartrate (BCDAT) and/or 0.5\% glucose during a 16-h photoperiod at $25 \mathrm{C}$ in 9-cm Petri dishes (Nishiyama et al. 2000). Growth rate measurements were taken at 30 day. The images of protonemal tissues and cells were obtained by a Microscope Digital Eyepiece DCM-510 attached to a Stemi 305 stereomicroscope (Zeiss, Germany) or Olympus CKX41 (Olympus, Japan) at 10 or 30 day. Seven-day-old protonema tissues grown in liquid BCDAT medium were used for proteomic, pull-down assay, co-immunoprecipitation and qRT-PCR analyses. The gametophores were grown on freeammonium tartrate BCD medium under the same conditions, and 8-week-old gametophores were used for the further analysis.

\section{Quantitative reverse transcription real-time PCR}

Total RNA from protonema and gametophores was isolated as previously described (Cove et al., 2009). RNA quality and quantity were evaluated via electrophoresis in an agarose gel with ethidium bromide staining. The concentration of total RNA was measured using a Quant-iTTM RNA Assay Kit (Thermo Fisher Scientific), 5-100 ng on a Qubit 3.0 (Invitrogen, US) fluorometer. The cDNA for qRT-PCR was synthesized using an MMLV RT Kit (Evrogen, Russia) according to the manufacturer's recommendations employing oligo(dT) 17 -primers from $2 \mu \mathrm{g}$ total RNA after DNase treatment. The primers were designed using Primer-BLAST (Ye et al., 2012) (Supplemental Table 4).

\section{Confirmation of FAMOSS transcription using nanopore RNA}

Nanopore data were downloaded from the BioProject with the accession number PRJNA681088 (Fesenko et al., 2021). Reads were mapped against the genome Physcomitrium 
510 patens V3.3 (Lang et al., 2018) by minimap 2.17 (Li, 2016) with the following parameters: -ax

511 splice -uf -k14 f -G2k. ONT reads with a primary alignment to the genome were retained. The

512 obtained 'SAM' files were sorted and indexed with 'SAMtools'(Li et al., 2009). Sashimi plot

513 was constructed in IGV v2.6.3 (Thorvaldsdottir et al., 2013).

\section{Generation of the overexpressing lines with FAMOSS-FLAG peptides}

The FAMOSS sequences fused with FLAG (DYKDDDDK) tag were obtained by PCR

with genomic DNA as a template and primers listed in the Supplemental Table 4. Amplicons were cloned into the pPLV27 vector (GenBank JF909480) using the ligation-independent cloning (LIC) procedure (Aslanidis and de Jong, 1990; De Rybel et al., 2011). The resulting plasmids - pPLV27-pep4-g4s-C-flag and pPLV27-N-flag-g4s-pep4 contain the C-terminus or the N-terminus FLAG-fused FAMOSS peptides respectively (Supplemental Figure 5). Before transformation, the plasmids were purified by Qiagen Plasmid Maxi Kit (Qiagen, Germany). Moss protoplasts were transformed with circular pPLV27-pep4-g4s-C-flag and pPLV27-N-flagg4s-pep4 plasmids. Mutant lines with the overexpression of fused FAMOSS-FLAG peptides were generated as described previously (Fesenko et al., 2019)

\section{CRISPR/Cas9-directed fusion with $\beta$-D-glucuronidase (GUS) protein}

The $\beta$-glucuronidase (GUS) reporter gene system was used to study spatial expression of FAMOSS peptide. The peptide coding sequence was used to search for CRISPR RNA (crRNA)

530 for cloning. The mutant lines with FAMOSS coding sequence fused with GUS reporter protein were created using the CRISPR/Cas9 system and the pTZ-donor4 plasmid (Supplemental Methods). Protoplasts were transformed with circular sgRNA, pTZ-donor4 and pBRF as previously described (Fesenko et al., 2019).

\section{GUS activity assay}

Histochemical GUS staining was carried out using $\beta$-Glucuronidase Reporter Gene

536 Staining Kit (Sigma-Aldrich) according to the manufacturer's recommendations. Moss tissues 537 were vacuum-infiltrated with Staining Solution for 2 minutes and incubated at $37^{\circ} \mathrm{C}$ overnight $(>$ 53816 hours). Stained samples were imaged by Microscope Digital Eyepiece DCM-510 attached to 
539 a Stemi 305 stereomicroscope (Zeiss, Germany) or Olympus CKX41 (Olympus, Japan) in the 540 bright-field.

\section{The conservation analysis}

To identify the FAMOSS orthologs in different plant taxa, TBLASTN search with default parameters was performed using CNGBdb BLAST Service for 1000 Plants (oneKP or 1KP)

544 trancriptomes (https://db.cngb.org/onekp/). The alignments were filtered by $E$-value $<10^{-3}$ cut-off 545 and manually curated.

547 (Katoh and Standley, 2013) and transformed into an HMM profile by hmmbuild tool from 548 HMMER v3.3.2 package (Eddy, 2011). Orfipy tool (Singh and Wurtele, 2021) was used to 549 predicted proteins (> 30 aa) from the transcriptomes of conifers and eudicots species that were 550 downloaded from 551 https://datacommons.cyverse.org/browse/iplant/home/shared/commons_repo/curated/oneKP_cap 552 stone_2019. Hmmsearch tool (hammer.org, $E<0.01$ ) was used to identify possible FAMOSS 553 orthologs in this protein dataset and obtained hits were manually curated.

\section{Sequence Alignment and Conserved Motif Identification}

The full amino acid sequences of the identified FAMOSS orthologs were extracted from the corresponding transcripts and used for multiple alignments using MAFFT v.7 with default settings. The resulting multiple sequence alignment (MSA) was inspected and adjusted manually. The online programs MEME (Multiple Expectation Maximization for Motif Elicitation; https://meme-suite.org/meme) with default parameter settings were used to search for conserved motifs.

\section{The phylogenetic analysis of the FAMOSS orthologs}

We reconstructed a maximum-likelihood (ML) phylogenetic tree based on the entire MSA of 250 orthologs using IQ-TREE v.1.6.12 (Nguyen et al., 2015) with autodetected models

564 (JTTDCMut+G4 model). Extremely divergent sequences were excluded from the analysis. The phylogenetic trees were generated by the Toytree package (Eaton et al., 2020). 


\section{Prediction of FAMOSS 3D-structure}

In order to predict FAMOSS peptide 3-D structure we used AlphaFold2 (Jumper et al., 2021) algorithm, which was run as Google Colaboratory project on GitHub with custom multiple sequence alignment (MSA) option. For MSA construction for a particular peptide we firstly obtained a list of 100 mostly similar sequences using BLASTP search against a file containing possible FAMOSS homologs. We then performed multiple protein alignment with MCOFFEE online tool with default parameters (http://tcoffee.crg.cat/apps/tcoffee/do:mcoffee (Notredame et al., 2000). Resulting MSA .fas file was used in the algorithm with default $\begin{array}{llllll}\text { parameters. } & \text { Structures } & \text { were } & \text { visualised } & \text { with }\end{array}$ (https://github.com/schrodinger/pymol-open-source).

\section{LC-MS/MS analysis and protein identifications}

Quantitative proteomic analysis was conducted as described previously (Spechenkova et al., 2021). Additional details are described in the Supplemental Methods. Briefly, proteins were extracted by phenol extraction method (Faurobert et al., 2007), digested by $1 \mu \mathrm{g}$ sequence-grade modified trypsin (Promega, Madison, WI, USA) at $37{ }^{\circ} \mathrm{C}$ overnight, iTRAQ labelling (Applied Biosystems, Foster City, CA, USA) was conducted according to the manufacturer's manual. Proteins were labelled with the iTRAQ tags as follows: Wild type - 113, 114, 115 isobaric tags, FAMOSS OE and KO - 116, 117, 118 ones. The samples after pull-down assay and co-immunoprecipitation were also digested by $1 \mu \mathrm{g}$ sequence-grade modified trypsin (Promega, Madison, WI, USA) at $37{ }^{\circ} \mathrm{C}$ overnight in the same manner, but were not labeled by iTRAQ labels. LC-MS/MS analysis was carried out on an Ultimate 3000 RSLCnano HPLC system connected to a QExactive Plus mass spectrometer (Thermo Fisher Scientific, USA). Tandem mass spectra were analysed by PEAKS Studio version 8.0 software (Bioinformatics Solutions Inc., Waterloo, Canada). The custom database was built from Phytozome database $P$. patens combined with chloroplast and mitochondrial proteins (33,053 records).

\section{Pull-down assay}

The plasmids pET-mSAV and pET-mSAV-pep4 were generated based on pET-plasmid (Novagen, USA) and contained sequence coding for mature streptavidinstreptavidine (SAV) fused with the sequences for TEV-proteinase site (pET-mSAV) or the same sequences fused with and FAMOSS peptide (pET-mSAV-pep4). The E. coli Rosetta 2 (DE3) strain was used to 
596 obtain recombinant proteins. Expression was induced by adding IPTG to a final concentration of

$5970,5 \mathrm{mM}$. The cells were disrupted by sonication using a Branson Sonifier 250 (VWR Scientific,

598 USA) sonicator according to the manufacturer's instructions. The lysate was purified by 599 centrifugation $(15,000 \times \mathrm{g}, 25 \mathrm{~min})$ and washed twice with $1 \%(\mathrm{v} / \mathrm{v})$ Triton $\mathrm{X}-100$. The pellet was 600 dissolved in $6 \mathrm{M}$ guanidine chloride, $\mathrm{pH} 1,5$, dialysed against $200 \mathrm{mM} \mathrm{NaHCO} 3 \mathrm{pH} 8,0$ and 601 purified using iminobiotin agarose (Thermo Fisher Scientific, USA) according to manufacturer's 602 instructions.

To the tissue of wild type protonema, grounded in liquid nitrogen, the lysis buffer (PBS, $150 \mathrm{mM} \mathrm{NaCl}, 0,1 \%$ Triton X-100, 0,2\% NP-40, Protease inhibitor cocktail for plant cell extracts (Sigma)) was added. Lysate was incubated on ice for $30 \mathrm{~min}$ with rotation (Heidolph Duomax 1030) and centrifuged $14000 \mathrm{~g}, 15 \mathrm{~min}, 4 \mathrm{C}$ (Eppendorf Centrifuge $5418 \mathrm{R}) .15 \mu \mathrm{g}$ of recombinant FAMOSS-streptavidin/streptavidin was added to supernatant and incubated $1 \mathrm{~h}$ on ice with rotation. Proteins in solution were cross-linked by $3 \mathrm{mM}$ DSP (Thermo Fisher Scientific Pierce) and incubated during $2 \mathrm{~h}$. Reaction was quenched by $40 \mathrm{mM}$ Tris- $\mathrm{HCl} \mathrm{pH} 8$ during 15 min on ice, then $50 \mathrm{mM}$ ammonium bicarbonate was added. Samples were centrifuged at 14000

$611 \mathrm{~g} 10 \mathrm{~min}$. The $20 \mathrm{mkl}$ of pre-washed by washing buffer $(50 \mathrm{mM}$ ammonium bicarbonate- $\mathrm{NaOH}$

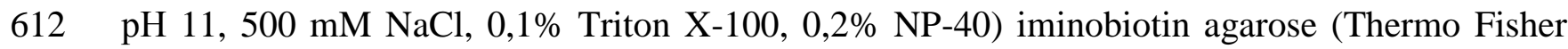

613 Scientific Pierce) was added to supernatant and incubated on ice during $1 \mathrm{~h}$, washed 4 times by washing buffer. Proteins of interest were eluted three times by $50 \mathrm{mM}$ ammonium acetate $\mathrm{pH} 3$ with $500 \mathrm{mM} \mathrm{NaCl}$. Eluates was neutralised by $20 \mathrm{mM}$ Tris and clean-uped by Amicon Ultra- 0.5

616 Centrifugal Filter Unit Ultracel-3K (Millipore). After that the crosslinker was cleaved with 20 $617 \mathrm{mM}$ DTT by heating at $50 \mathrm{C}$.

\section{Co-immunoprecipitation}

To identify the proteins which interact with FAMOSS peptide the co-immunoprecipitation approach was used. The plant tissue, which was grounded at liquid nitrogen, was homogenized in $450 \mathrm{mkl}$ lysis buffer $(50 \mathrm{mM}$ Tris- $\mathrm{HCl} \mathrm{pH} \mathrm{8,150} \mathrm{mM} \mathrm{NaCl,} \mathrm{0,1 \%} \mathrm{Tween-20,} 10 \mathrm{mkl} / \mathrm{ml}$ protease inhibitor cocktail (Sigma)). The resulting suspension was incubated on ice 30 min with rotation and centrifuged at $13000 \mathrm{~g}$ for $15 \mathrm{~min}, 4 \mathrm{C}$ (Eppendorf Centrifuge $5418 \mathrm{R}$ ). Proteins in

624 the clear supernatant (protein extract) were cross-linked with 5 mM DSP (Thermo Fisher 625 Scientific Pierce) for $2 \mathrm{~h}$ with agitation. The reaction was stopped with $40 \mathrm{mM}$ Tris- $\mathrm{HCl} \mathrm{pH} 8$ 626 during $15 \mathrm{~min}$ incubation. Samples were centrifuged at $14000 \mathrm{~g} 10 \mathrm{~min}$. The $50 \mu \mathrm{l}$ pre-washed 627 with washing buffer (50 mM Tris- $\mathrm{HCl} \mathrm{pH} \mathrm{8,} 150 \mathrm{mM} \mathrm{NaCl}, 0,1 \%$ Tween-20) M2 anti-FLAG 
628 magnetic beads (Sigma) was added to supernatant. The samples were incubated $2 \mathrm{~h}$ on ice with 629 rotation of the washing buffer and once with $700 \mathrm{mkl}$ of TBS $(50 \mathrm{mM}$ Tris- $\mathrm{HCl} \mathrm{pH} 8,150 \mathrm{mM}$

$630 \mathrm{NaCl}$ ). The protein complexes were specifically eluted with $125 \mathrm{mkl}$ of $100 \mathrm{ng} / \mathrm{mkl} 3 x$ FLAG 631 peptide (Sigma) solution in TBS during $30 \mathrm{~min}$ on-ice incubation with agitation. The crosslinker 632 was cleaved with $20 \mathrm{mM}$ DTT by heating at $50 \mathrm{C}$.

\section{SDS-PAGE and in-gel trypsin digestion}

Proteins after pull-down and Co-IP experiments were vacuum dried, dissolved in a sodium dodecyl sulphate-polyacrylamide gel electrophoresis (SDS-PAGE) loading buffer (Bio-Rad) and separated on $15 \%$ SDS-PAGE gels following the Laemmli method (Laemmli, 1970). Gels were stained as described previously (Schagger, 2006). The gel pieces corresponding $25 \mathrm{kDa} \mathrm{MW}$ were cut, destained by $15 \mathrm{mM}$ sodium thiosulfate and $50 \mathrm{mM}$ potassium ferricyanide. Protein ingel trypsin digestion was conducted as described by (Rubtsova et al., 2018).

\section{Regeneration of protonema from protoplasts} and incubated during the 6 days on solid BCD agar medium. Regenerated protonema was

643 detected by microscopy analysis.

\section{Fluorescent microscopy}

To detect vesicles, protonemal cells were stained by the $5 \mu \mathrm{M}$ of fluorescent dye

646 SynaptoGreen C4 (equivalent to FM1-43, Biotium, USA. SynaptoGreen C4 is a synonym of 647 fluorescent probe which is originally called FM1-43 dye and is available from Biotium under the 648 trademark name of SynaptoGreen ${ }^{\mathrm{TM}}$ ) during $5 \mathrm{~min}$ and washed. To visualize cell walls the 649 protonema cells were stained by $10 \mu \mathrm{g} / \mathrm{mL}$ of propidium iodide (fluorescein diacetate, Sigma650 Aldrich, USA) during 5 min. Fluorescence was detected by Axio Imager M2 microscope (Zeiss) 651 with an AxioCam 506 mono digital camera (Zeiss) and Zen 2.6 pro software (Zeiss). Filter unit 65244 FITC ( $\lambda$ ex BP $475 \mathrm{~nm} / 40 \mathrm{~nm}$; $\lambda$ em BP $530 \mathrm{~nm} / 50 \mathrm{~nm}$ ) was used for SynaptoGreen C4 653 fluorescence detection, Filter unit 20 Rhodamin ( $\lambda$ ex BP 546/12 nm; $\lambda$ em BP 575-640 nm) was used 654 for propidium iodide. 


\section{Statistical analysis}

656 Statistical analysis and visualisation were made in Python v. 3.7.5(Van Rossum, Drake,

657 1995) using modules scipy 1.5.2 (Virtanen et al., 2020), seaborn 0.11.1 (Waskom, 2021), numpy

658 1.20.1, pandas 1.2. 3 (McKinney, 2012).

$659 \quad$ Data availability

660 The mass spectrometry proteomic data have been deposited to the ProteomeXchange

661 Consortium via the PRIDE (Perez-Riverol et al., 2019) partner repository with the dataset

662 identifiers PXD028296 and PXD028342.

663 


\section{REFERENCES}

665

666

667

668

669

670

671

672

673

674

675

676

677

678

679

680

681

682

683

684

685

686

687

688

689

690

691

692

693

694

695

696

697

1. Anderson, D.M., Anderson, K.M., Chang, C.L., Makarewich, C.A., Nelson, B.R., McAnally, J.R., Kasaragod, P., Shelton, J.M., Liou, J., Bassel-Duby, R., et al. (2015). A Micropeptide Encoded by a Putative Long Noncoding RNA Regulates Muscle Performance. Cell 160:595-606. 10.1016/j.cell.2015.01.009.

2. Aslanidis, C., and de Jong, P.J. (1990). Ligation-independent cloning of PCR products (LIC-PCR). Nucleic Acids Res 18:6069-6074. 10.1093/nar/18.20.6069.

3. Bailey, T.L., Johnson, J., Grant, C.E., and Noble, W.S. (2015). The MEME Suite. Nucleic Acids Res 43:W39-49. 10.1093/nar/gkv416.

4. Blanvillain, R., Young, B., Cai, Y.M., Hecht, V., Varoquaux, F., Delorme, V., Lancelin, J.M., Delseny, M., and Gallois, P. (2011). The Arabidopsis peptide kiss of death is an inducer of programmed cell death. Embo J 30:1173-1183. 10.1038/emboj.2011.14.

5. Bove, J., Vaillancourt, B., Kroeger, J., Hepler, P.K., Wiseman, P.W., Geitmann A. (2008) Magnitude and Direction of Vesicle Dynamics in Growing Pollen Tubes Using Spatiotemporal Image Correlation Spectroscopy and Fluorescence Recovery after Photobleaching. Plant

Physiology 147:1646-1658. https://doi.org/10.1104/pp.108.120212

6. Brito, M.S., DePaoli, H.C., Cossalter, V., Avanci, N.C., Ferreira, P.B., Azevedo, M.S., Strini, E.J., Quiapim, A.C., Goldman, G.H., Peres, L.E.P., et al. (2018). A novel cysteinerich peptide regulates cell expansion in the tobacco pistil and influences its final size. Plant science : an international journal of experimental plant biology 277:55-67. 10.1016/j.plantsci.2018.09.002.

7. Casson, S.A., Chilley, P.M., Topping, J.F., Evans, I.M., Souter, M.A., and Lindsey, K. (2002). The POLARIS gene of Arabidopsis encodes a predicted peptide required for correct root growth and leaf vascular patterning. The Plant cell 14:1705-1721. $10.1105 /$ tpc.002618.

8. Castro, A., Vidal, S., and Ponce de Leon, I. (2016). Moss Pathogenesis-Related-10 Protein Enhances Resistance to Pythium irregulare in Physcomitrella patens and Arabidopsis thaliana. Front Plant Sci 7:580. 10.3389/fpls.2016.00580.

9. Chen, C., Heo, J.B. (2018) Overexpression of Constitutively Active OsRab11 in Plants Enhances Tolerance to High Salinity Levels. J. Plant Biol. 61:169-176. 10.1007/s12374018-0048-0.

10. Chen, D., He, L., Lin, M., Jing, Y., Liang, C., Liu, H., Gao, J., Zhang, W., and Wang, M. (2021). A ras-related small GTP-binding protein, RabE1c, regulates stomatal movements 
698

699

700

701

702

703

704

705

706

707

708

709

710

711

712

713

714

715

716

717

718

719

720

721

722

723

724

725

726

727

728

729

730

731

and drought stress responses by mediating the interaction with $\mathrm{ABA}$ receptors. Plant science : an international journal of experimental plant biology 306:110858. 10.1016/j.plantsci.2021.110858.

11. Chen, Q.J., Deng, B.H., Gao, J., Zhao, Z.Y., Chen, Z.L., Song, S.R., Wang, L., Zhao, L.P., Xu, W.P., Zhang, C.X., et al. (2020). A miRNA-Encoded Small Peptide, vvimiPEP171d1, Regulates Adventitious Root Formation. Plant Physiol 183:656-670. 10.1104/pp.20.00197.

12. Chng, S.C., Ho, L.N., Tian, J., and Reversade, B. (2013). ELABELA: A Hormone Essential for Heart Development Signals via the Apelin Receptor. Dev Cell 27:672-680. 10.1016/j.devcel.2013.11.002.

13. Chugunova, A., Loseva, E., Mazin, P., Mitina, A., Navalayeu, T., Bilan, D., Vishnyakova, P., Marey, M., Golovina, A., Serebryakova, M., et al. (2019). LINC00116 codes for a mitochondrial peptide linking respiration and lipid metabolism. Proc Natl Acad Sci U S A 116:4940-4945. 10.1073/pnas.1809105116.

14. Collonnier, C., Guyon-Debast, A., Maclot, F., Mara, K., Charlot, F., and Nogue, F. (2017). Towards mastering CRISPR-induced gene knock-in in plants: Survey of key features and focus on the model Physcomitrella patens. Methods 121-122:103-117. 10.1016/j.ymeth.2017.04.024.

15. Cove, D.J., Perroud, P.F., Charron, A.J., McDaniel, S.F., Khandelwal, A., and Quatrano, R.S. (2009). Isolation of DNA, RNA, and protein from the moss Physcomitrella patens gametophytes. Cold Spring Harb Protoc 2009:pdb prot5146. 10.1101/pdb.prot5146.

16. D'Lima, N.G., Ma, J., Winkler, L., Chu, Q., Loh, K.H., Corpuz, E.O., Budnik, B.A., Lykke-Andersen, J., Saghatelian, A., and Slavoff, S.A. (2017). A human microprotein that interacts with the mRNA decapping complex. Nat Chem Biol 13:174-180. 10.1038/Nchembio.2249.

17. Dao, T.T., Linthorst, H.J., and Verpoorte, R. (2011). Chalcone synthase and its functions in plant resistance. Phytochemistry reviews : proceedings of the Phytochemical Society of Europe 10:397-412. 10.1007/s11101-011-9211-7.

18. De Rybel, B., van den Berg, W., Lokerse, A., Liao, C.Y., van Mourik, H., Moller, B., Peris, C.L., and Weijers, D. (2011). A versatile set of ligation-independent cloning vectors for functional studies in plants. Plant Physiol 156:1292-1299. 10.1104/pp.111.177337.

19. Delwiche, C.F., and Cooper, E.D. (2015). The Evolutionary Origin of a Terrestrial Flora. Current biology : CB 25:R899-910. 10.1016/j.cub.2015.08.029. 
20. Dong, X., Wang, D., Liu, P., Li, C., Zhao, Q., Zhu, D., and Yu, J. (2013). Zm908p11, encoded by a short open reading frame (sORF) gene, functions in pollen tube growth as a profilin ligand in maize. J Exp Bot 64:2359-2372. 10.1093/jxb/ert093.

21. de Graaf, B.H.J., Cheung, A.Y., Andreyeva, T., Levasseur, K., Kieliszewski, M., Wu H. (2005) Rab11 GTPase-Regulated Membrane Trafficking Is Crucial for Tip-Focused Pollen Tube Growth in Tobacco. The Plant Cell 17: 2564-2579. 10.1105/tpc.105.033183.

22. Eaton D.A.R. (2020) Toytree: A minimalist tree visualization and manipulation library for Python. Methods Ecol Evol. 11:187-191. 10.1111/2041-210X.13313.

23. Eddy, S.R. (2011). Accelerated Profile HMM Searches. PLoS Comput Biol 7:e1002195. 10.1371/journal.pcbi.1002195.

24. Elliott, L., Moore, I., Kirchhelle, C. (2020) Spatio-temporal control of post-Golgi exocytic trafficking in plants. J Cell Sci 13: jcs237065. https://doi.org/10.1242/jcs.237065

25. Faurobert, M., Pelpoir, E., and Chaib, J. (2007). Phenol extraction of proteins for proteomic studies of recalcitrant plant tissues. Methods Mol Biol 355:9-14. 10.1385/159745-227-0:9.

26. Fesenko I., Shabalina, S.A., Mamaeva, A., Knyazev, A., Glushkevich, A., Lyapina, I., Ziganshin, R., Kovalchuk, S., Kharlampieva, D., Lazarev, V., Taliansky, M., Koonin, E.V. (2021) A vast pool of lineage-specific microproteins encoded by long non-coding RNAs in plants. Nucleic Acids Research, https://doi.org/10.1093/nar/gkab816

27. Fesenko, I., Kirov, I., Kniazev, A., Khazigaleeva, R., Lazarev, V., Kharlampieva, D., Grafskaia, E., Zgoda, V., Butenko, I., Arapidi, G., et al. (2019). Distinct types of short open reading frames are translated in plant cells. Genome Res 29:1464-1477. 10.1101/gr.253302.119.

28. Fesenko, I.A., Arapidi, G.P., Skripnikov, A.Y., Alexeev, D.G., Kostryukova, E.S., Manolov, A.I., Altukhov, I.A., Khazigaleeva, R.A., Seredina, A.V., Kovalchuk, S.I., et al. (2015). Specific pools of endogenous peptides are present in gametophore, protonema, and protoplast cells of the moss Physcomitrella patens. Bmc Plant Biol 15:87. 10.1186/s12870-015-0468-7.

29. Fujimoto, M., and Tsutsumi, N. (2014). Dynamin-related proteins in plant post-Golgi traffic. Front Plant Sci 5:408. 10.3389/fpls.2014.00408. green plant genomics. Nucleic Acids Res 40:D1178-1186. 10.1093/nar/gkr944. 
31. Guo, B., Wu, S., Zhu, X., Zhang, L., Deng, J., Li, F., Wang, Y., Zhang, S., Wu, R., Lu, J., et al. (2020). Micropeptide CIP2A-BP encoded by LINC00665 inhibits triple-negative breast cancer progression. Embo J 39:e102190. 10.15252/embj.2019102190.

32. Hanada, K., Higuchi-Takeuchi, M., Okamoto, M., Yoshizumi, T., Shimizu, M., Nakaminami, K., Nishi, R., Ohashi, C., Iida, K., Tanaka, M., et al. (2013). Small open reading frames associated with morphogenesis are hidden in plant genomes. Proceedings of the National Academy of Sciences of the United States of America 110:2395-2400. 10.1073/pnas.1213958110.

33. Hoepflinger, M.C., Geretschlaeger, A., Sommer, A., Hoeftberger, M., Nishiyama, T., Sakayama, H., Hammerl, P., Tenhaken, R., Ueda, T., and Foissner, I. (2013). Molecular and biochemical analysis of the first ARA6 homologue, a RAB5 GTPase, from green algae. J Exp Bot 64:5553-5568. 10.1093/jxb/ert322.

34. Huang, J.Z., Chen, M., Chen, D., Gao, X.C., Zhu, S., Huang, H.Y., Hu, M., Zhu, H.F., and Yan, G.R. (2017). A Peptide Encoded by a Putative lncRNA HOXB-AS3 Suppresses Colon Cancer Growth. Mol Cell 68:171-+. 10.1016/j.molcel.2017.09.015.

35. Ikeuchi, M., Yamaguchi, T., Kazama, T., Ito, T., Horiguchi, G., and Tsukaya, H. (2011). ROTUNDIFOLIA4 regulates cell proliferation along the body axis in Arabidopsis shoot. Plant Cell Physiol 52:59-69. 10.1093/pcp/pcq138.

36. Immarigeon, C., Frei, Y., Delbare, S.Y.N., Gligorov, D., Almeida, P.M., Grey, J., Fabbro, L., Nagoshi, E., Billeter, J.C., Wolfner, M.F., et al. (2021). Identification of a micropeptide and multiple secondary cell genes that modulate Drosophila male reproductive success. P Natl Acad Sci USA 118ARTN e2001897118

37. 10.1073/pnas.2001897118.

38. Jaeger, R., and Moody, L.A. (2021). A fundamental developmental transition in Physcomitrium patens is regulated by evolutionarily conserved mechanisms. Evolution \& development 23:123-136. 10.1111/ede.12376.

39. Jang, G., and Dolan, L. (2011). Auxin promotes the transition from chloronema to caulonema in moss protonema by positively regulating PpRSL1and PpRSL2 in Physcomitrella patens. The New phytologist 192:319-327. 10.1111/j.14698137.2011.03805.x.

40. Jelinkova, A., Malinska, K., Simon, S., Kleine-Vehn, J., Parezova, M., Pejchar, P., Kubes, M., Martinec, J., Friml, J., Zazimalova, E., et al. (2010). Probing plant membranes with FM dyes: tracking, dragging or blocking? The Plant journal : for cell and molecular biology 61:883-892. 10.1111/j.1365-313X.2009.04102.x. 
41. Jilly, R., Khan, N.Z., Aronsson, H., and Schneider, D. (2018). Dynamin-Like Proteins Are Potentially Involved in Membrane Dynamics within Chloroplasts and Cyanobacteria. Front Plant Sci 9:206. 10.3389/fpls.2018.00206.

42. Jumper, J., Evans, R., Pritzel, A., Green, T., Figurnov, M., Ronneberger, O., Tunyasuvunakool, K., Bates, R., Zidek, A., Potapenko, A., et al. (2021). Highly accurate protein structure prediction with AlphaFold. Nature 596:583-589. 10.1038/s41586-02103819-2.

43. Katoh, K., and Standley, D.M. (2013). MAFFT multiple sequence alignment software version 7: improvements in performance and usability. Mol Biol Evol 30:772-780. 10.1093/molbev/mst010.

44. Krogh, A., Larsson, B., von Heijne, G., and Sonnhammer, E.L. (2001). Predicting transmembrane protein topology with a hidden Markov model: application to complete genomes. Journal of molecular biology 305:567-580. 10.1006/jmbi.2000.4315.

45. Kwon, C., Lee, J.H., and Yun, H.S. (2020). SNAREs in Plant Biotic and Abiotic Stress Responses. Molecules and cells 43:501-508. 10.14348/molcells.2020.0007.

46. Laemmli, U.K. (1970). Cleavage of structural proteins during the assembly of the head of bacteriophage T4. Nature 227:680-685. 10.1038/227680a0.

47. Lang, D., Ullrich, K.K., Murat, F., Fuchs, J., Jenkins, J., Haas, F.B., Piednoel, M., Gundlach, H., Van Bel, M., Meyberg, R., et al. (2018). The Physcomitrella patens chromosome-scale assembly reveals moss genome structure and evolution. The Plant journal : for cell and molecular biology 93:515-533. 10.1111/tpj.13801.

48. Li, H. (2016). Minimap and miniasm: fast mapping and de novo assembly for noisy long sequences. Bioinformatics 32:2103-2110. 10.1093/bioinformatics/btw152.

49. Li, H., Handsaker, B., Wysoker, A., Fennell, T., Ruan, J., Homer, N., Marth, G., Abecasis, G., Durbin, R., and Genome Project Data Processing, S. (2009). The Sequence Alignment/Map format and SAMtools. Bioinformatics 25:2078-2079. 10.1093/bioinformatics/btp352. RNAs: Focus on lncRNAs, pri-miRNAs, and circRNAs. Exp Cell Res 361:1-8. 10.1016/j.yexcr.2017.10.010.

51. Lycett, G. (2008). The role of Rab GTPases in cell wall metabolism. J Exp Bot 59:40614074. 10.1093/jxb/ern255. Growth Regul 26:369-388.10.1007/s00344-007-9022-7. 
53. Magny, E.G., Pueyo, J.I., Pearl, F.M.G., Cespedes, M.A., Niven, J.E., Bishop, S.A., and Couso, J.P. (2013). Conserved Regulation of Cardiac Calcium Uptake by Peptides Encoded in Small Open Reading Frames. Science 341:1116-1120. 10.1126/science. 1238802 .

54. MartiniEre, A., and Moreau, P. (2020). Complex roles of Rabs and SNAREs in the secretory pathway and plant development: a never-ending story. J Microsc 280:140-157. 10.1111/jmi.12952.

55. Matsumoto, A., Pasut, A., Matsumoto, M., Yamashita, R., Fung, J., Monteleone, E., Saghatelian, A., Nakayama, K.I., Clohessy, J.G., and Pandolfi, P.P. (2017). mTORC1 and muscle regeneration are regulated by the LINC00961-encoded SPAR polypeptide. Nature 541:228-232. 10.1038/nature21034.

56. McKinney,W. (2012) Python for Data Analysis: Data Wrangling with Pandas, NumPy, and IPython 'O'Reilly Media, Inc.'.

57. Mellacheruvu, D., Wright, Z., Couzens, A.L., Lambert, J.P., St-Denis, N.A., Li, T., Miteva, Y.V., Hauri, S., Sardiu, M.E., Low, T.Y., et al. (2013). The CRAPome: a contaminant repository for affinity purification-mass spectrometry data. Nature methods 10:730-736. 10.1038/nmeth.2557.

58. Minamino, N., and Ueda, T. (2019). RAB GTPases and their effectors in plant endosomal transport. Current opinion in plant biology 52:61-68. 10.1016/j.pbi.2019.07.007.

59. Nguyen, L.T., Schmidt, H.A., von Haeseler, A., and Minh, B.Q. (2015). IQ-TREE: a fast and effective stochastic algorithm for estimating maximum-likelihood phylogenies. Mol Biol Evol 32:268-274. 10.1093/molbev/msu300.

60. Nishiyama, T., Sakayama, H., de Vries, J., Buschmann, H., Saint-Marcoux, D., Ullrich, K.K., Haas, F.B., Vanderstraeten, L., Becker, D., Lang, D., et al. (2018). The Chara Genome: Secondary Complexity and Implications for Plant Terrestrialization. Cell 174:448-464 e424. 10.1016/j.cell.2018.06.033. et al. (2020). A micropeptide encoded by lncRNA MIR155HG suppresses autoimmune inflammation via modulating antigen presentation. Science advances 6:eaaz2059. 10.1126/sciadv.aaz2059.

62. Notredame, C., Higgins, D.G., and Heringa, J. (2000). T-Coffee: A novel method for fast and accurate multiple sequence alignment. Journal of molecular biology 302:205-217. 10.1006/jmbi.2000.4042.

63. One Thousand Plant Transcriptomes, I. (2019). One thousand plant transcriptomes and the phylogenomics of green plants. Nature 574:679-685. 10.1038/s41586-019-1693-2. 
869

870

871

872

873

874

875

876

877

878

879

880

881

882

883

884

885

886

887

888

889

890

891

892

893

894

895

896

897

898

899

900

901

64. Orr, R.G., Cheng, X., Vidali, L., and Bezanilla, M. (2020). Orchestrating cell morphology from the inside out - using polarized cell expansion in plants as a model. Current opinion in cell biology 62:46-53. 10.1016/j.ceb.2019.08.004.

65. Orr, R.G., Furt, F., Warner, E.L., Agar, E.M., Garbarino, J.M., Cabral, S.E., Dubuke, M.L., Butt, A.M., Munson, M., and Vidali, L. (2021). Rab-E and its interaction with myosin XI are essential for polarised cell growth. The New phytologist 229:1924-1936. 10.1111/nph.17023.

66. Ovecka, M., Berson, T., Beck, M., Derksen, J., Samaj, J., Baluska, F., and Lichtscheidl, I.K. (2010). Structural sterols are involved in both the initiation and tip growth of root hairs in Arabidopsis thaliana. The Plant cell 22:2999-3019. 10.1105/tpc.109.069880.

67. Ow, S.Y., Salim, M., Noirel, J., Evans, C., Rehman, I., and Wright, P.C. (2009). iTRAQ underestimation in simple and complex mixtures: "the good, the bad and the ugly". J Proteome Res 8:5347-5355. 10.1021/pr900634c.

68. Paniagua, C., Bilkova, A., Jackson, P., Dabravolski, S., Riber, W., Didi, V., Houser, J., Gigli-Bisceglia, N., Wimmerova, M., Budinska, E., et al. (2017). Dirigent proteins in plants: modulating cell wall metabolism during abiotic and biotic stress exposure. J Exp Bot 68:3287-3301. 10.1093/jxb/erx 141.

69. Peaucelle, A., Braybrook, S.A., Le Guillou, L., Bron, E., Kuhlemeier, C., and Hofte, H. (2011). Pectin-induced changes in cell wall mechanics underlie organ initiation in Arabidopsis. Current biology : CB 21:1720-1726. 10.1016/j.cub.2011.08.057.

70. Perez-Riverol, Y., Csordas, A., Bai, J., Bernal-Llinares, M., Hewapathirana, S., Kundu, D.J., Inuganti, A., Griss, J., Mayer, G., Eisenacher, M., et al. (2019) The PRIDE database and related tools and resources in 2019: improving support for quantification data. Nucleic Acids Res. 47: D442-D450.

71. Pleskot, R., Pejchar, P., Bezvoda, R., Lichtscheidl, I.K., Wolters-Arts, M., Marc, J., Žárský, V., Potocký, M. (2012). Turnover of phosphatidic acid through distinct signaling pathways affects multiple aspects of pollen tube growth in tobacco. Frontiers in plant science 3:1-13.

72. Polycarpou-Schwarz, M., Gross, M., Mestdagh, P., Schott, J., Grund, S.E., Hildenbrand, C., Rom, J., Aulmann, S., Sinn, H.P., Vandesompele, J., et al. (2018). The cancerassociated microprotein CASIMO1 controls cell proliferation and interacts with squalene epoxidase modulating lipid droplet formation. Oncogene 37:4750-4768. 10.1038/s41388018-0281-5. 
902

903

904

905

906

907

908

909

910

911

912

913

914

915

916

917

918

919

920

921

922

923

924

925

926

927

928

929

930

931

932

933

934

935

73. Preuss, M.L., Serna, J., Falbel, T.G., Bednarek, S.Y., and Nielsen, E. (2004). The Arabidopsis Rab GTPase RabA4b localizes to the tips of growing root hair cells. The Plant cell 16:1589-1603. 10.1105/tpc.021634.

74. Rawat, A., Brejskova, L., Hala, M., Cvrckova, F., and Zarsky, V. (2017). The Physcomitrella patens exocyst subunit EXO70.3d has distinct roles in growth and development, and is essential for completion of the moss life cycle. The New phytologist 216:438-454. 10.1111/nph.14548.

75. Rensing, S.A., Goffinet, B., Meyberg, R., Wu, S.Z., and Bezanilla, M. (2020). The Moss Physcomitrium (Physcomitrella) patens: A Model Organism for Non-Seed Plants. The Plant cell 32:1361-1376. 10.1105/tpc.19.00828.

76. Rohrig, H., Schmidt, J., Miklashevichs, E., Schell, J., and John, M. (2002). Soybean ENOD40 encodes two peptides that bind to sucrose synthase. Proc Natl Acad Sci U S A 99:1915-1920. 10.1073/pnas.022664799.

77. Rounds, C.M., and Bezanilla, M. (2013). Growth mechanisms in tip-growing plant cells. Annual review of plant biology 64:243-265. 10.1146/annurev-arplant-050312-120150.

78. Rubtsova, M., Naraykina, Y., Vasilkova, D., Meerson, M., Zvereva, M., Prassolov, V., Lazarev, V., Manuvera, V., Kovalchuk, S., Anikanov, N., et al. (2018). Protein encoded in human telomerase RNA is involved in cell protective pathways. Nucleic Acids Res 46:8966-8977. 10.1093/nar/gky705.

79. Sabovljevic, M., Vujicic, M., Sabovljevic A. (2014). Plant growth regulators in bryophytes. Botanica serbica 38:99-107.

80. Samaj, J., Read, N.D., Volkmann, D., Menzel, D., Baluška, F. (2005) The endocytic network in plants. Trends in Cell Biology 15: 425-433. https://doi.org/10.1016/j.tcb.2005.06.006

81. Schagger, H. (2006). Tricine-SDS-PAGE. Nat Protoc 1:16-22. 10.1038/nprot.2006.4.

82. Singh, A., Vivek, A.T., and Kumar, S. (2021). AlnC: An extensive database of long noncoding RNAs in angiosperms. Plos One 16:e0247215. 10.1371/journal.pone.0247215.

83. Singh, U., and Wurtele, E.S. (2021). orfipy: a fast and flexible tool for extracting ORFs. Bioinformatics 10.1093/bioinformatics/btab090.

84. Slavoff, S.A., Heo, J.H., Budnik, B.A., Hanakahi, L.A., and Saghatelian, A. (2014). A Human Short Open Reading Frame ( sORF)-encoded Polypeptide That Stimulates DNA End Joining. J Biol Chem 289:10950-10957. 10.1074/jbc.C113.533968.

85. Spechenkova, N., Fesenko, I.A., Mamaeva, A., Suprunova, T.P., Kalinina, N.O., Love, A.J., and Taliansky, M. (2021). The Resistance Responses of Potato Plants to Potato 
Virus Y Are Associated with an Increased Cellular Methionine Content and an Altered SAM:SAH Methylation Index. Viruses 1310.3390/v13060955.

938

939

940

941

942

943

944

945

946

947

948

949

950

951

952

953

954

955

956

957

958

959

960

961

962

963

964

965

966

967

968

969

86. Steger, M., Diez, F., Dhekne, H.S., Lis, P., Nirujogi, R.S., Karayel, O., Tonelli, F., Martinez, T.N., Lorentzen, E., Pfeffer, S.R., Alessi D.R., Mann, M. (2017) Systematic proteomic analysis of LRRK2-mediated Rab GTPase phosphorylation establishes a connection to ciliogenesis. eLife 6:e31012 DOI: 10.7554/eLife.31012

87. Stenzel, I., Hause, B., Miersch, O., Kurz, T., Maucher, H., Weichert, H., Ziegler, J., Feussner, I., and Wasternack, C. (2003). Jasmonate biosynthesis and the allene oxide cyclase family of Arabidopsis thaliana. Plant Mol Biol 51:895-911. 10.1023/a:1023049319723.

88. Stevenson, S.R., Kamisugi, Y., Trinh, C.H., Schmutz, J., Jenkins, J.W., Grimwood, J., Muchero, W., Tuskan, G.A., Rensing, S.A., Lang, D., et al. (2016). Genetic Analysis of Physcomitrella patens Identifies ABSCISIC ACID NON-RESPONSIVE, a Regulator of ABA Responses Unique to Basal Land Plants and Required for Desiccation Tolerance. The Plant cell 28:1310-1327. 10.1105/tpc.16.00091.

89. Szczesniak, M.W., Rosikiewicz, W., and Makalowska, I. (2016). CANTATAdb: A Collection of Plant Long Non-Coding RNAs. Plant Cell Physiol 57:e8. 10.1093/pcp/pcv201.

90. Szumlanski, A.L., and Nielsen, E. (2009). The Rab GTPase RabA4d regulates pollen tube tip growth in Arabidopsis thaliana. The Plant cell 21:526-544. 10.1105/tpc.108.060277.

91. Tavormina, P., De Coninck, B., Nikonorova, N., De Smet, I., and Cammue, B.P. (2015). The Plant Peptidome: An Expanding Repertoire of Structural Features and Biological Functions. The Plant cell 27:2095-2118. 10.1105/tpc.15.00440.

92. Thelander, M., Landberg, K., and Sundberg, E. (2018). Auxin-mediated developmental control in the moss Physcomitrella patens. J Exp Bot 69:277-290. 10.1093/jxb/erx255.

93. Thorvaldsdottir, H., Robinson, J.T., and Mesirov, J.P. (2013). Integrative Genomics Viewer (IGV): high-performance genomics data visualization and exploration. Brief Bioinform 14:178-192. 10.1093/bib/bbs017.

94. Tran, L.T., Taylor, J.S., and Constabel, C.P. (2012). The polyphenol oxidase gene family in land plants: Lineage-specific duplication and expansion. Bmc Genomics 13:395. 10.1186/1471-2164-13-395.

95. Vakirlis, N., Acar, O., Hsu, B., Castilho Coelho, N., Van Oss, S.B., Wacholder, A., Medetgul-Ernar, K., Bowman, R.W., 2nd, Hines, C.P., Iannotta, J., et al. (2020). De novo 
970

971

972

973

974

975

976

977

978

979

980

981

982

983

984

985

986

987

988

989

990

991

emergence of adaptive membrane proteins from thymine-rich genomic sequences. Nat Commun 11:781. 10.1038/s41467-020-14500-z.

96. van Gisbergen, P., Wu, S.-Z., Cheng, X., Pattavina, K.A., Bezanilla, M. (2020) In vivo analysis of formin dynamics in the moss P. patens reveals functional class diversification. J Cell Sci 133: jcs233791. https://doi.org/10.1242/jcs.233791

97. Van Rossum,G., Drake,F.L.,Jr (1995) Python tutorial Centrum voor Wiskunde en Informatica Amsterdam.

98. Vidali, L., and Bezanilla, M. (2012). Physcomitrella patens: a model for tip cell growth and differentiation. Current opinion in plant biology 15:625-631. 10.1016/j.pbi.2012.09.008.

99. Virtanen, P., Gommers, R., Oliphant, T.E., Haberland, M., Reddy, T., Cournapeau, D., Burovski, E., Peterson, P., Weckesser, W., Bright, J., et al. (2020). SciPy 1.0: fundamental algorithms for scientific computing in Python. Nature methods 17:261-272. 10.1038/s41592-019-0686-2.

100. Wang, X., Zhang, H., Gao, Y., Sun, G., Zhang, W., and Qiu, L. (2014). A comprehensive analysis of the Cupin gene family in soybean (Glycine max). Plos One 9:e110092. 10.1371/journal.pone.0110092.

101. Waskom,M. (2021) seaborn: statistical data visualization. J. Open Source Softw., 6:3021.

102. Ye, J., Coulouris, G., Zaretskaya, I., Cutcutache, I., Rozen, S., and Madden, T.L. (2012). Primer-BLAST: a tool to design target-specific primers for polymerase chain reaction. Bmc Bioinformatics 13:134. 10.1186/1471-2105-13-134. 


\section{Figure legends}

993 Figure 1. FAMOSS is a widely conserved plant smORF-encoded peptide. sequencing in Physcomitrium patens protonema and gametophores. B - Construction of FAMOSS coupled with a GUS reporter protein at the FAMOSS promoter. Black arrows indicate primers used for identification. C, D - GUS staining of the protonema $(\mathrm{C}$, scale bar $=$ $200 \mu \mathrm{m})$ and gametophores $(\mathrm{D}$, scale bar $=1 \mathrm{~mm})$. E - The alignment of the FAMOSS peptide and orthologous sequences from different plants. F - Phylogenomic tree of the identified FAMOSS orthologs. Mosses, burgundy; liverworts, blue; algae, red; ferns, gray; gymnosperms, orange; angiosperms, green. $\mathrm{G}$ - P. patens FAMOSS structure and structures of its orthologs, predicted using AlphaFold.

1003

Figure 2. The growth of mutant lines in different conditions.

A - The ratios of the two types of moss protonema filaments, caulonemata and chloronemata, in wild-type, FAMOSS-overexpressing (OE), and knockout (KO) plants. B - The lengths of the apical, first subapical, and second subapical cells. C - The effect of 6-benzylaminopurine (BAP) on protonemal morphology. Scale bar $=1 \mathrm{~mm} . \mathrm{D}-$ The effect of abscisic acid (ABA) on protonemal morphology. $\mathrm{E}$ - The effect of BAP on the protonemal growth rate. $\mathrm{F}$ - The effect of ABA on protonemal growth. $\mathrm{G}$ - The effect of medium composition on protonemal growth. $+\mathrm{G}+\mathrm{AT}, \mathrm{BCD}$ medium supplemented with glucose and ammonium tartrate; $+\mathrm{G}-\mathrm{AT}$, BCD medium with glucose but without ammonium tartrate. $\mathrm{H}$ - Fold change (FC) in FAMOSS transcription after treatments with ABA, BAP, and indole-3-acetic acid (IAA). I, J - Quantitative proteomic analysis of the FAMOSS-overexpressing (OE) and knockout (KO) plants. Volcano plots of the entire set of proteins quantified in the iTRAQ analysis in the FAMOSS OE (I) and KO (J) lines. Proteins with significant differences in abundance relative to the wild type are depicted in color. GSA, glutamate-1-semialdehyde 2,1-aminomutase; rbfA, ribosome-binding factor A; RPS20, ribosomal protein S20; RPS30, ribosomal protein S30; AOC, allene oxide cyclase; GST, glutathione $S$-transferase; CHS, chalcone synthase; $\mathrm{XTH}$, xyloglucan endotransglucosylase/hydrolase protein 23; PME, pectin methylesterase; rbcL, ribulose-1,5-bisphosphate carboxylase/oxygenase large subunit; GAPDH, glyceraldehyde-3-phosphate dehydrogenase A chloroplastic-like isoform X2; JAL3, jacalinrelated lectin 3; PPO, polyphenol oxidase; PAL, phenylalanine ammonia-lyase; PR10, pathogenesis-related protein; PR10-like, pathogenesis-related protein-like; DIR, dirigent protein; SNAP25, synaptosomal-associated protein 25; ASPL, tether containing UBX 
domain for GLUT4; DRP1, dynamin-related protein 1E; CLTA, clathrin light chain; PYL5, pyrobactin resistance 1-like 5; PMM, phosphomannomutase; SSU, ribulose-1,5-bisphosphate carboxylase/oxygenase small subunits; DUF1955, domain of unknown function. *, $P<0.05 ; * *, P<0.01 ; * * *, P<0.001$.

Figure 4. The growth rate of the mutant lines under stress-related conditions. A - Effect of $\mathrm{NaCl}$ on protonemal growth. $\mathrm{B}$ - Effect of paraquat (PQ) on protonemal growth. C - Effect of $\mathrm{NaCl}$ and $\mathrm{PQ}$ on protonemal morphology after 30 days of cultivation. D - FAMOSS expression under different stresses. $*, P<0.05 ; * * *, P<0.001$.

Figure 5. Analysis of the protein partners of the FAMOSS peptide. A - Diagram of the interactome experiments. B - Sequence and coverage of the FLAG-tagged FAMOSS sequence. C - Sequence of streptavidin (SAV)-tagged FAMOSS. D - Rab GTPases present in the two types of interactome experiments. Proteins detected in samples are marked with a plus sign, and those not detected are indicated with a minus sign. E - Alignment of Rab GTPases found in the FAMOSS interactomes.

Figure 6. The FAMOSS peptide affects vesicular trafficking. A-C - Apical protonemal cells stained with SynaptoGreen C4. (A - FAMOSS-overexpression line (OE); B - The wild type; C - FAMOSS knockout line (KO). D - Profile of SynaptoGreen C4 fluorescence in the apical cells. E - Boxplot showing the ratios of intensity of SynaptoGreen C4 staining at the distance $15 \mathrm{mkm}$ from the cell tip to the maximal intensity. ***, $P<0.001$. peptide. FAMOSS is expressed in favorable conditions and modulates the Rab signaling pathway to influence vesicular trafficking and ABA signaling. These processes increase protonemal growth, translation, and caulonemal formation, but decrease the ABA response and, thus, the adaptability to stresses. 
bioRxiv preprint doi: https://doi.org/10.1101/2021.1124 469821: this version posted November 25, 2021. The copyright holder for this

\section{Figures}

A

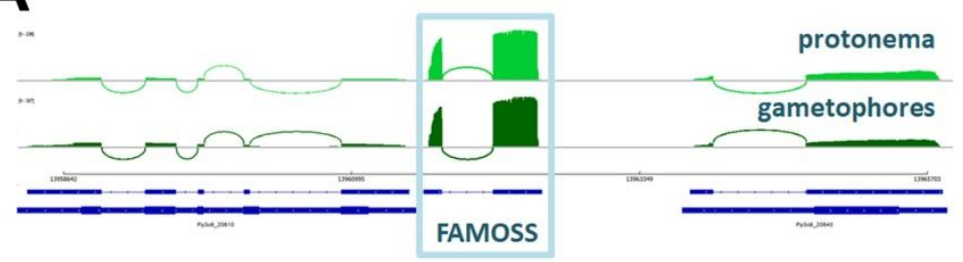

B

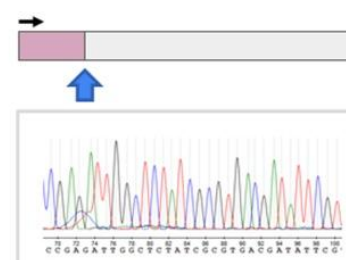

\begin{tabular}{|l|l} 
Genome & Plasmid left arm \\
\hline
\end{tabular}

E

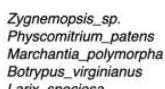
Larix_speciosa
Oenothera_gaura

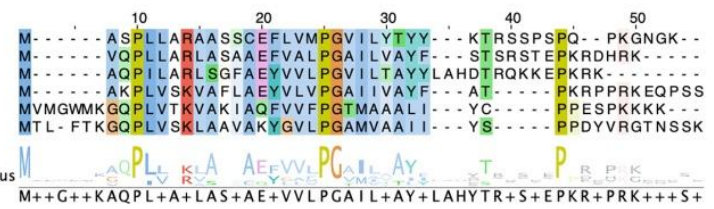

$\mathbf{F}$

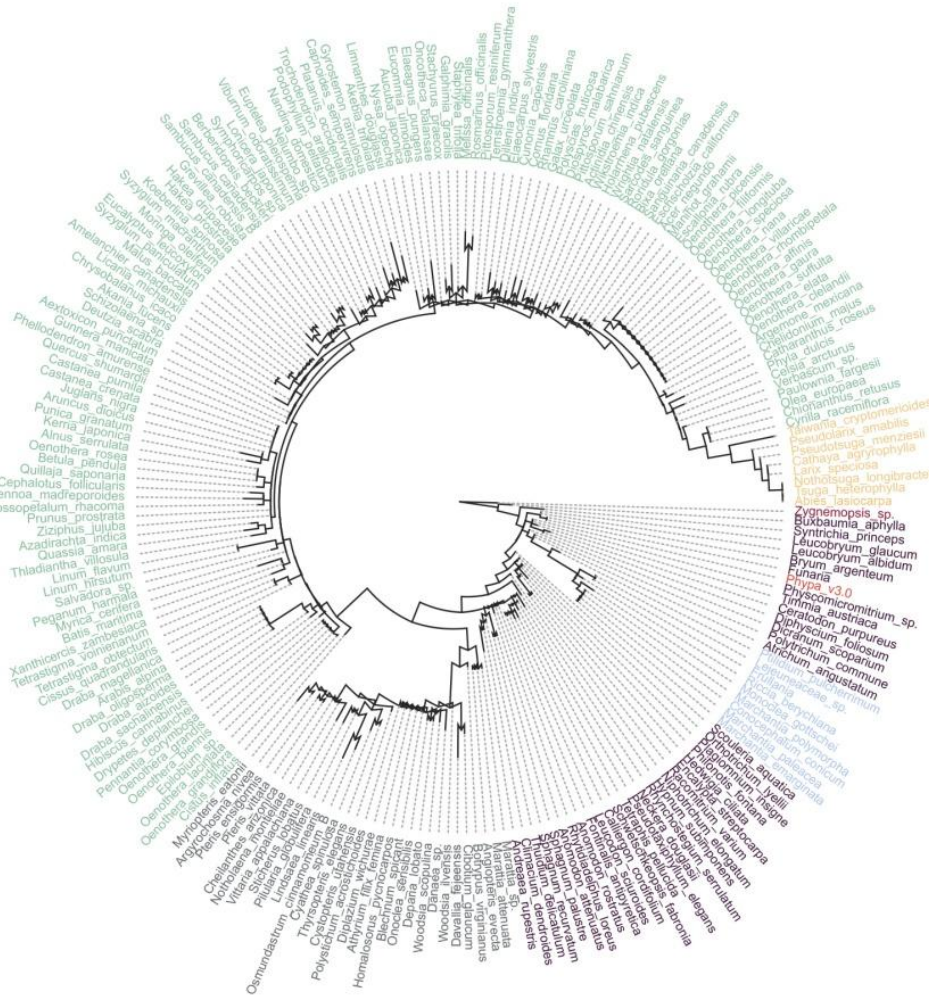

C
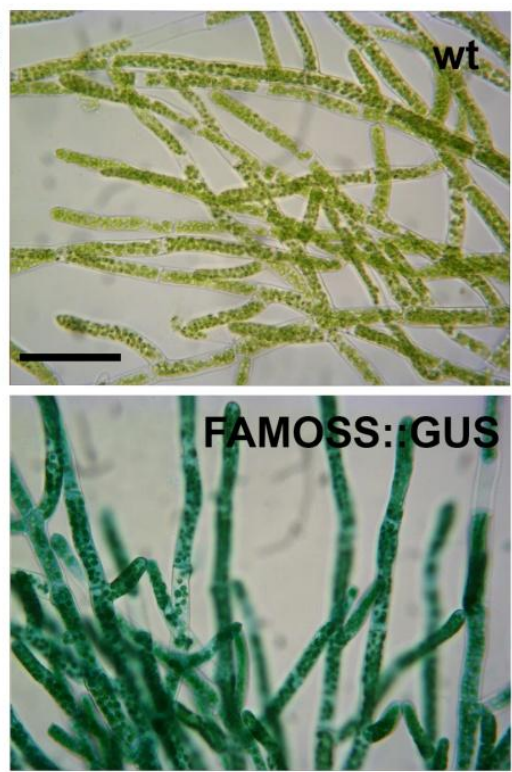

D

FAMOSS::GUS

G

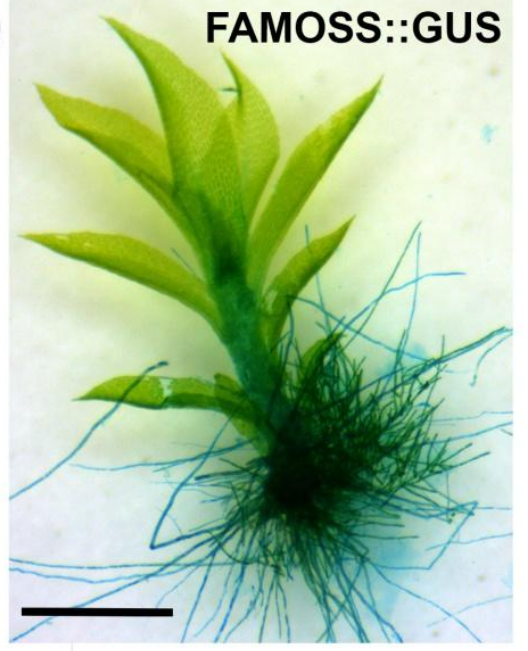

Physcomitrium patens

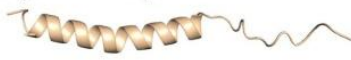

Zygnemopsis sp.

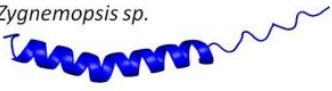

Marchantia polymorpha cogocerror

Cheilanthes arizonica

vecosos?

Larix speciosa

Betula pendula

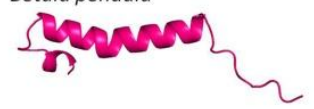


bioRxiv preprint doi: https://doi.org/10.1101/2021.11.24.469821; this version posted November 25, 2021. The copyright holder for this preprint (which was not certified by peer review) is the author/funder, who has granted bioRxiv a license to display the preprint in perpetuity. It is made available under aCC-BY-NC-ND 4.0 International license.

$\mathbf{A}$

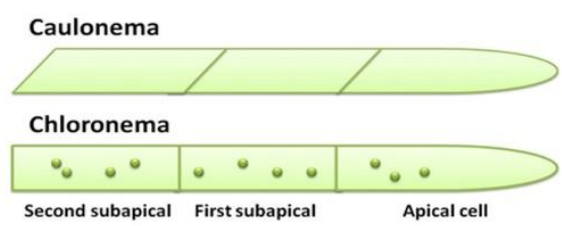

Type \%, caulonema \%, chloronema Ratio Caul/Chlor

\begin{tabular}{l|l|l|l|}
\hline WT & $59.72 \%$ & $40.28 \%$ & 1.5 \\
\hline OE & $76.73 \%$ & $23.27 \%$ & 3.3 \\
\hline KO & $33.48 \%$ & $66.52 \%$ & 0.5 \\
\hline
\end{tabular}

C

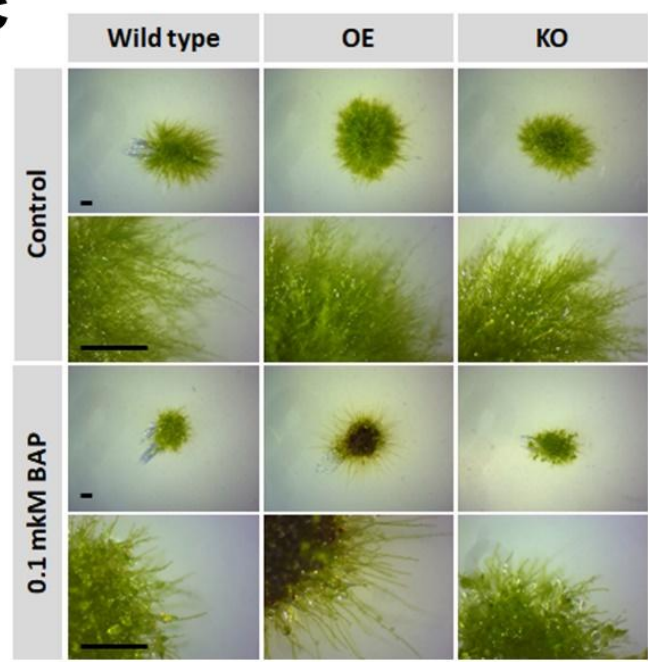

$\mathbf{E}$

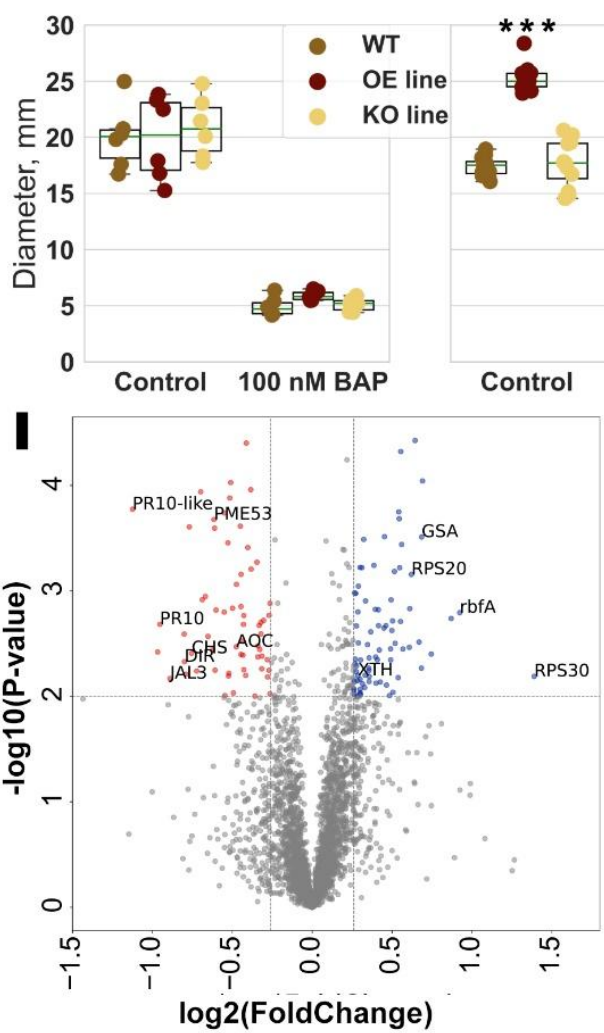

B

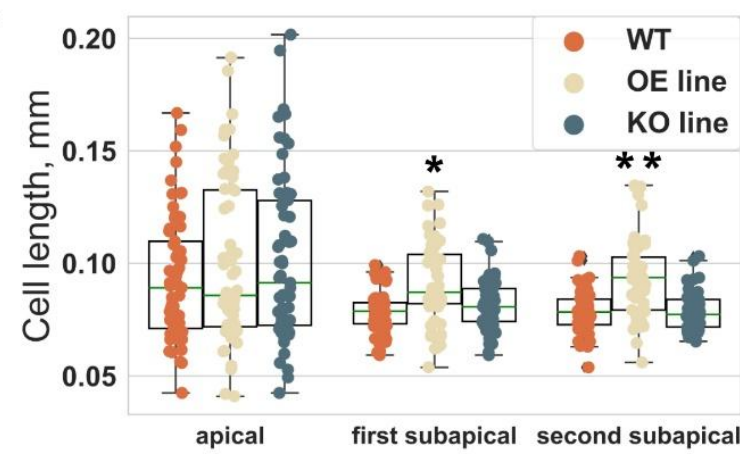

D

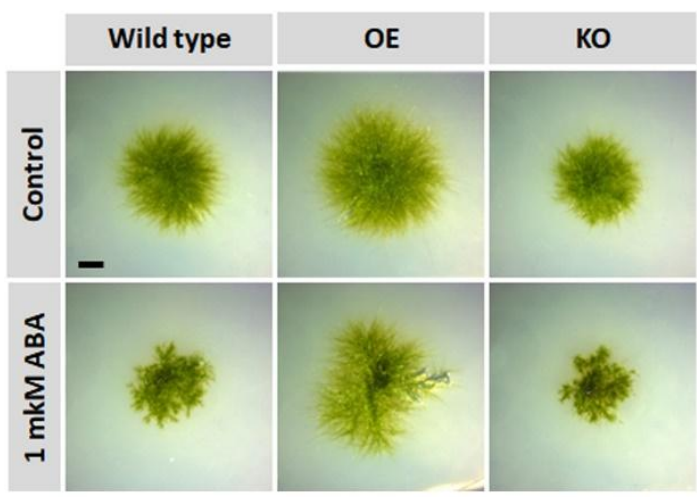

F

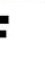

G

H
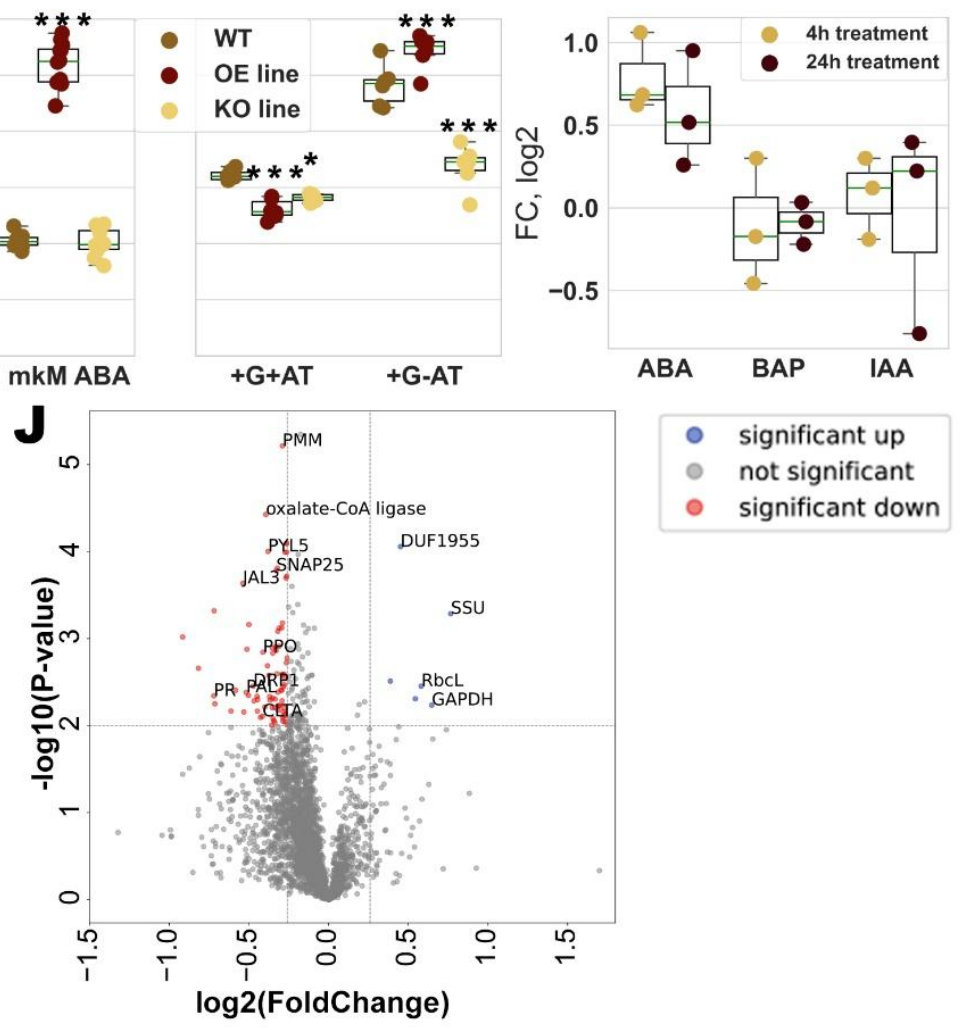

- significant up

- not significant

- significant down 
A

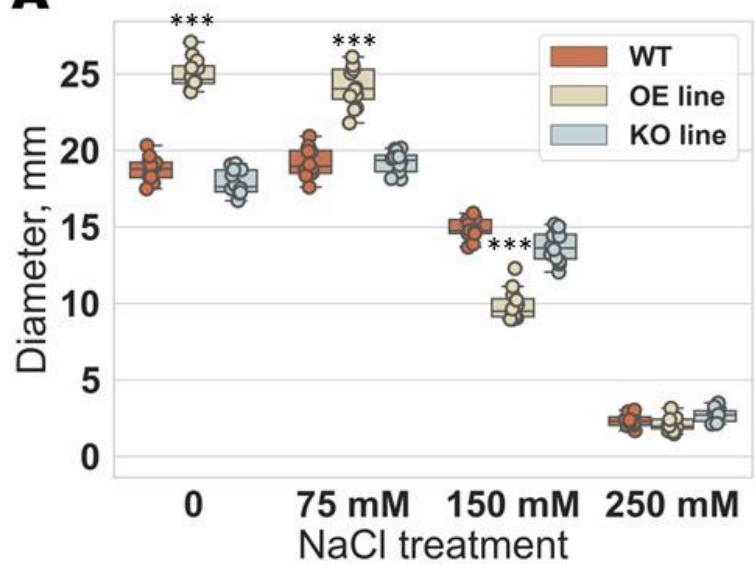

C
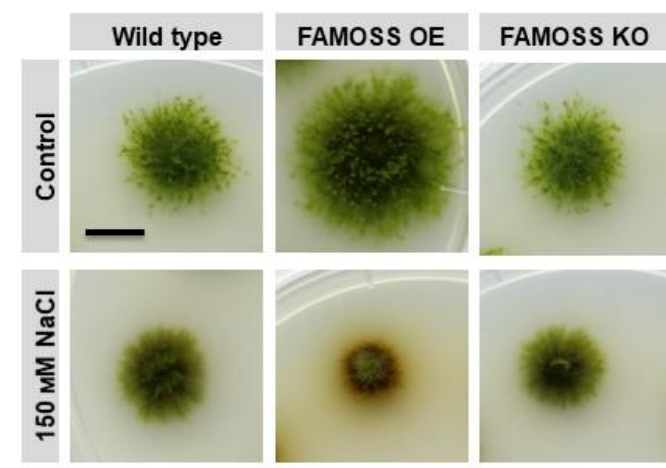

1059

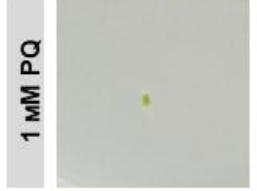

B

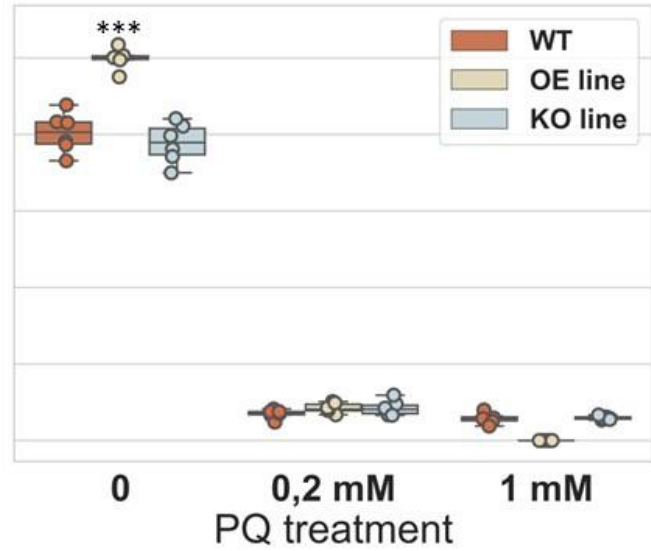

D

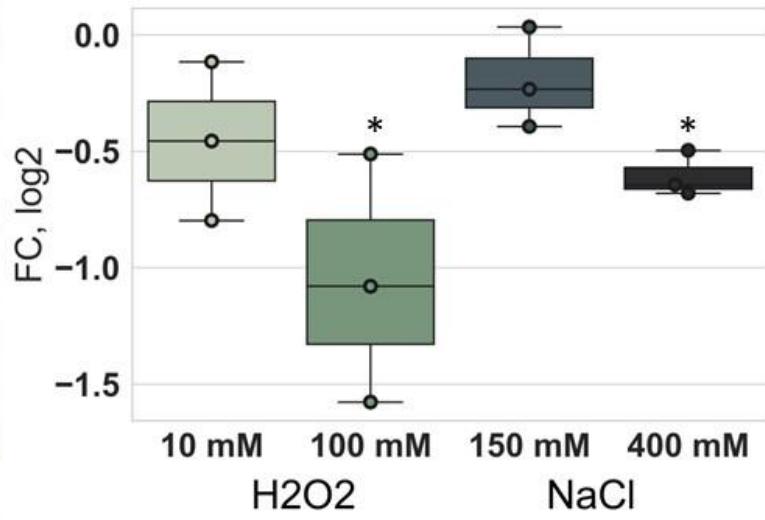

Figure 3. 
A

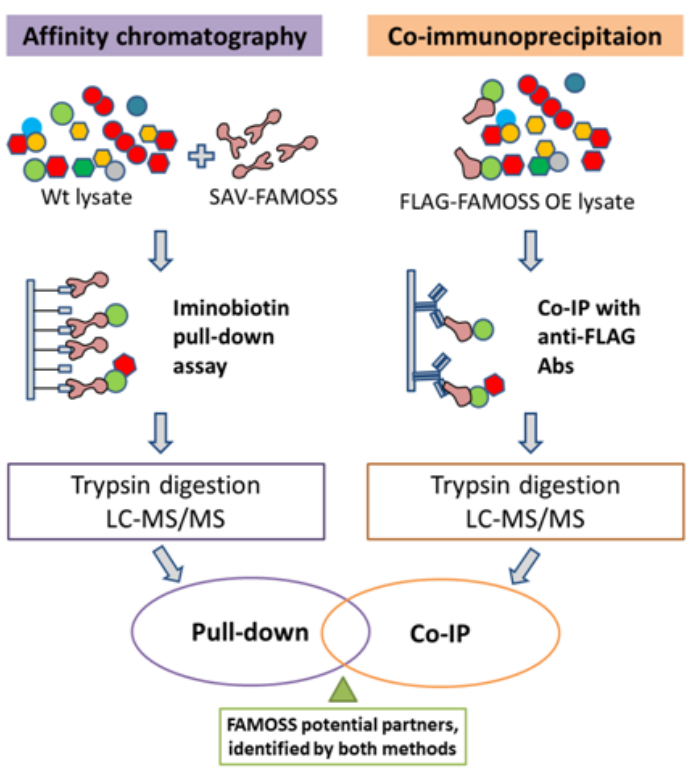

B FAMOSS with FLAG-tag on the $\mathrm{N}$-end 1. MDYKDDDDKG GGGSGGGGMV QPLLARLASA AEFVALPGAI LVAYFSTSRS TEPKRDHRK

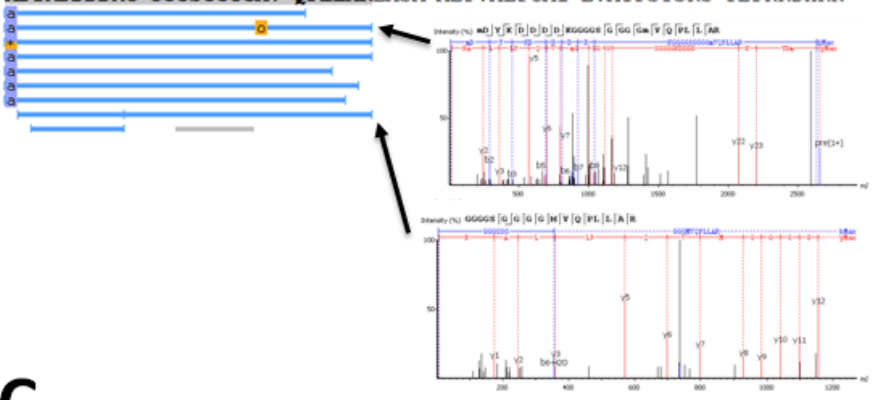

FAMOSS-SAV

MGSMRKIVVAAIAVSLTTVSITASASADPSKDSKAQVSAAEAGITGTMYN QLGSTIIVTAGADGALTGTYESAVGNAESRYVLTGRYDSAPATDGSGTA LGWTVAWKNNYRNAHSATTWSGQYVG GAEARINTQWLLTSGTTEANA WKSTLVGHDTFTKVKPSAASIDAAKKAGVNNGNPLDAVQQSRGGSGG GSENLYFQGVDMVQPLLARLASAAEFVALPGAILVAYFSTSRSTEPKRD HRK

D

\begin{tabular}{|ccc|}
\hline Suloclass of Rab GTPases & Pulldown with FAMOSS-SAV & Coimmunoprecipitation \\
\hline RabD2B/Rab-1A & + & + \\
RabA2B/Rab-11A & + & + \\
RabF/Rab-5 & + & - \\
RabA5A/Rab-11A & + & + \\
RabE/Rab-8A & + & - \\
RabG/Rab-7A & + & + \\
\hline
\end{tabular}

E

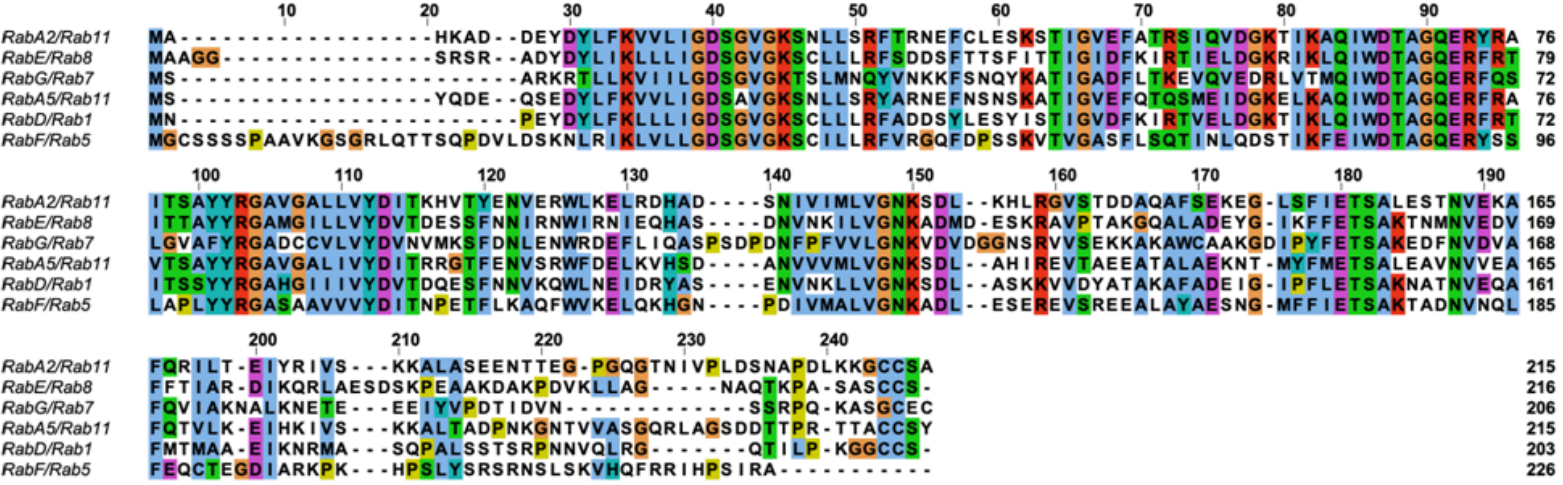


bioRxiv preprint doi: https://doi.org/10.1101/2021.11.24.469821; this version posted November 25, 2021. The copyright holder for this preprint (which was not certified by peer review) is the author/funder, who has granted bioRxiv a license to display the preprint in perpetuity. It is made available under aCC-BY-NC-ND 4.0 International license.
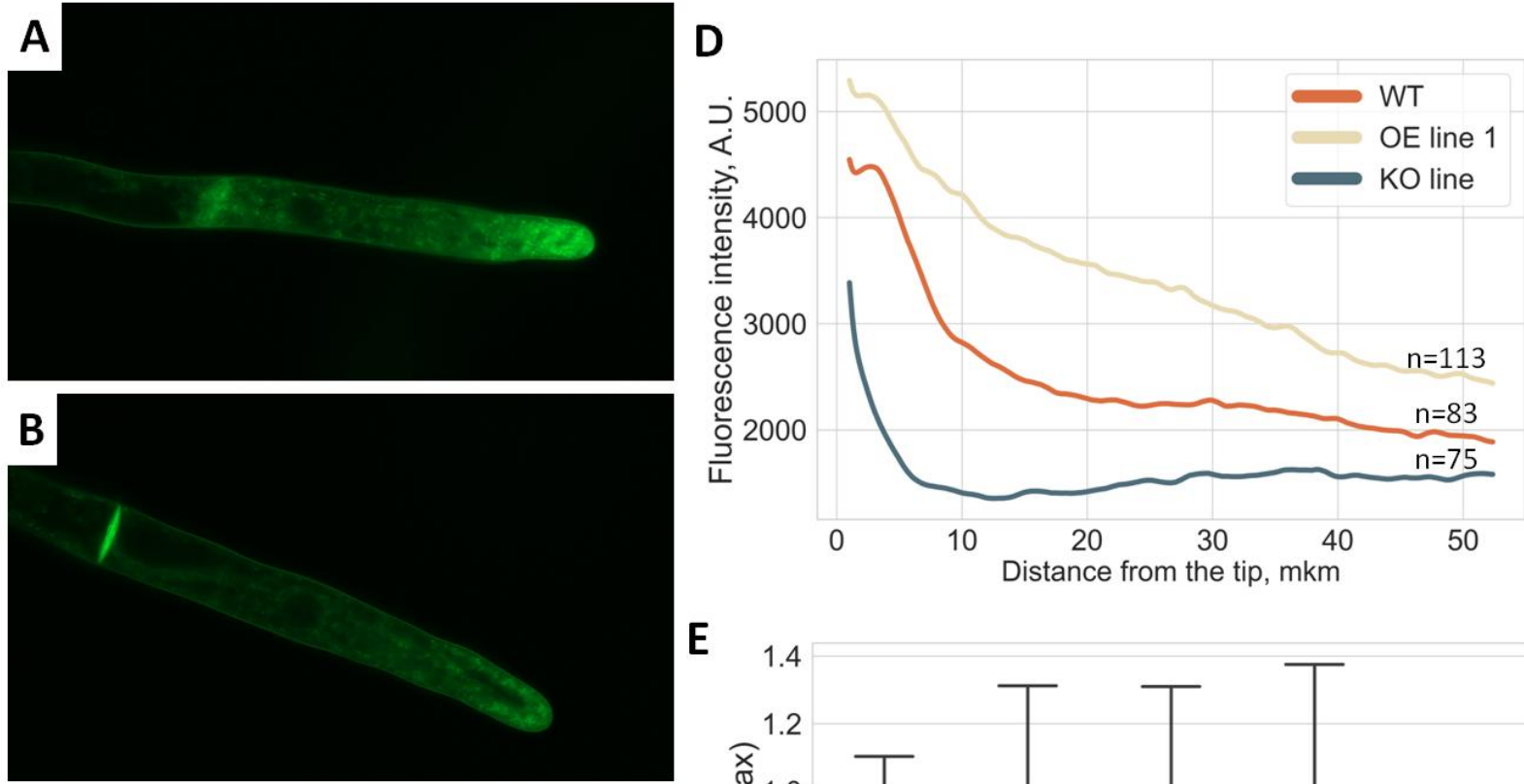

E
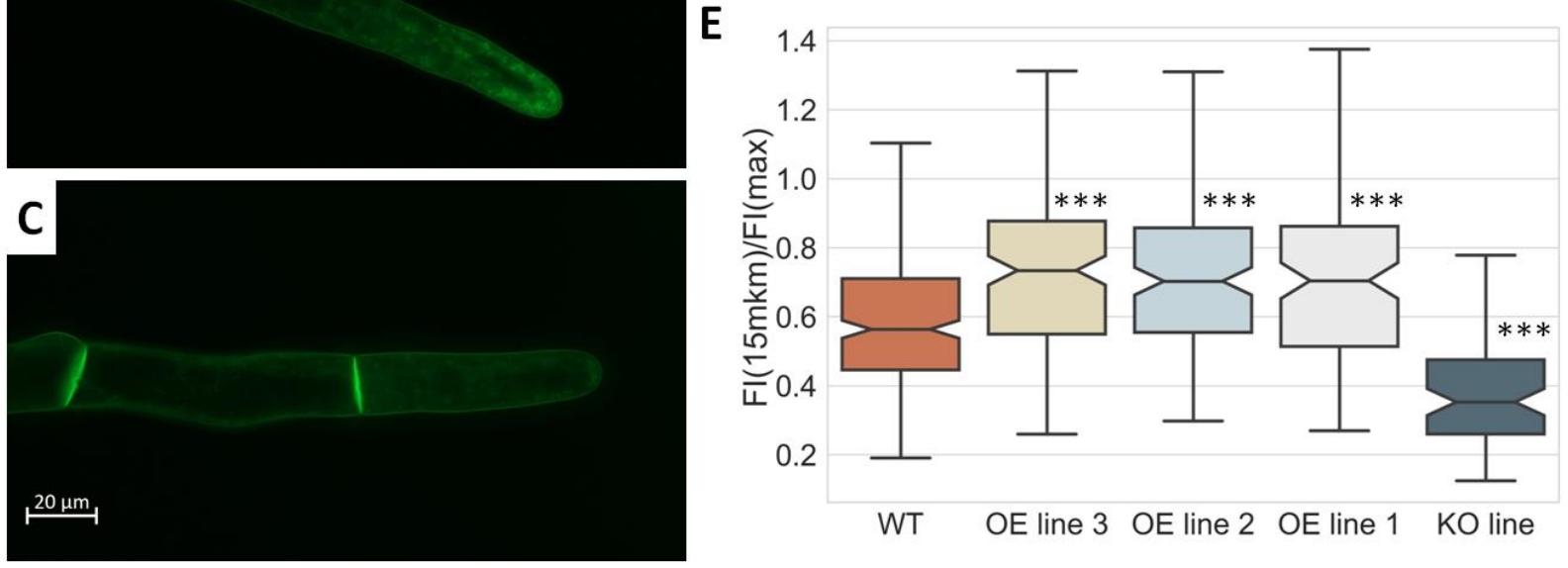

Figure 5. 